Revue internationale P.M.E.

Économie et gestion de la petite et moyenne entreprise

Revue

internationale

PME

\title{
L'acquisition des ressources humaines en PME
}

\section{Bruno Fabi et Denis J. Garand}

Volume 6, numéro 3-4, 1994

URI : https://id.erudit.org/iderudit/1008233ar

DOI : https://doi.org/10.7202/1008233ar

Aller au sommaire du numéro

Éditeur(s)

Presses de l’Université du Québec

ISSN

0776-5436 (imprimé)

1918-9699 (numérique)

Découvrir la revue

Citer cet article

Fabi, B. \& Garand, D. J. (1994). L'acquisition des ressources humaines en PME. Revue internationale P.M.E., 6(3-4), 91-129. https://doi.org/10.7202/1008233ar

\section{Résumé de l'article}

Cet article résume une démarche exploratoire visant à relever les principales pratiques d'acquisition des ressources humaines (RH) en contexte de PME. Pour y parvenir, l'expérience internationale a été analysée à travers la documentation empirique rapportant des résultats de terrain dans divers pays d'Europe et d'Amérique du Nord. Une première constatation révèle que très peu de PME nord-américaines effectuent une réelle planification des $\mathrm{RH}$ ou une véritable analyse des emplois. Certaines établissent un plan de recrutement à court terme et rédigent des descriptions de tâches, mais ces outils ne leur servent qu'à se conformer à des normes externes. La situation diffère toutefois en France et dans les pays où ces pratiques sont réglementées ou encouragées. En outre, la majorité des PME démontrent peu d'imagination dans le choix des sources de recrutement et se bornent à quelques méthodes passives. La sélection se limite à l'analyse des formulaires de demande d'emploi et à l'entrevue individuelle; les critères de sélection et les tests apparaissent fortement sous-utilisés; et l'accueil ne dépasse guère la présentation aux collègues, exception faite des PME plus participatives. Enfin, l'analyse du degré de formalisation des pratiques d'acquisition des RH fait ressortir la nécessité de réorienter rétude de la GRH en favorisant la discussion sur les implications éventuelles d'une gestion renouvelée dans les organisations, particulièrement dans les PME.
Ce document est protégé par la loi sur le droit d'auteur. L'utilisation des services d'Érudit (y compris la reproduction) est assujettie à sa politique d'utilisation que vous pouvez consulter en ligne.

https://apropos.erudit.org/fr/usagers/politique-dutilisation/ 


\title{
L'acquisition des ressources humaines en PME
}

\author{
Bruno FABI* \\ Université du Québec à Trois-Rivières \\ Denis J. GARAND** \\ Université du Québec en Abitibi-Témiscamingue
}

\begin{abstract}
RÉSUMÉ
Cet article résume une démarche exploratoire visant à relever les principales pratiques d'acquisition des ressources humaines $(R H)$ en contexte de PME. Pour y parvenir, l'expérience internationale a été analysée à travers la documentation empirique rapportant des résultats de terrain dans divers pays d'Europe et d'Amérique du Nord. Une première constatation révèle que très peu de PME nord-américaines effectuent une réelle planification des $R H$ ou une véritable analyse des emplois. Certaines établissent un plan de recrutement à court terme et rédigent des descriptions de tâches, mais ces outils ne leur servent qu'à se conformer à des normes externes. La situation diffère toutefois en France et dans les pays où ces pratiques sont réglemen-
\end{abstract}

* Bruno Fabi est professeur agrégé à l'UQTR et coordonne les cours de GRH aux premier et deuxième cycles. Il est titulaire d'un doctorat (D. Ps.) en psychologie industrielle et organisationnelle de l'Université de Montréal. Il est l'auteur de plusieurs ouvrages, articles scientifiques et professionnels. Ses intérêts de recherche et d'intervention portent principalement sur la GRH, la gestion participative et le développement organisationnel. Membre du GREPME, il agit également à titre d'évaluateur pour divers périodiques et organismes subventionnaires. Adresse : Département d'administration et d'économique, Université du Québec à Trois-Rivières, C.P. 500, Trois-Rivières (Québec) G9A 5 H7.

** Denis J. Garand est chargé d'enseignement en management à l'Université du Québec en Abitibi-Témiscamingue (Rouyn-Noranda - UQAT). Il détient une maîtrise de recherche (M. Sc.) en gestion de PME de l'UQTR et a collaboré à plusieurs projets de recherche appliqués aux petites entreprises. Chercheur indépendant en GRH, en gestion de PME ainsi qu'en gestion de projets, ses travaux récents portent sur les pratiques de GRH des PME, leur degré de formalisation et l'impact de certains facteurs de contingence : taille organisationnelle, propriétairedirigeant, vision entrepreneuriale, présence syndicale, orientations stratégiques. Adresse : 371, av. Bellevue, Laval (Québec) H7C 1T4. 
tées ou encouragées. En outre, la majorité des PME démontrent peu d'imagination dans le choix des sources de recrutement et se bornent à quelques méthodes passives. La sélection se limite à l'analyse des formulaires de demande d'emploi et à l'entrevue individuelle; les critères de sélection et les tests apparaissent fortement sous-utilisés; et l'accueil ne dépasse guère la présentation aux collègues, exception faite des PME plus participatives. Enfin, l'analyse du degré de formalisation des pratiques d'acquisition des $R H$ fait ressortir la nécessité de réorienter l'étude de la GRH en favorisant la discussion sur les implications éventuelles d'une gestion renouvelée dans les organisations, particulièrement dans les PME.

\section{ABSTRACT}

This paper summarizes an exploratory analysis of the major employment and placement activities of small and medium-sized businesses. Much of the international data on human resources (HR) in small firms has been reviewed, based on empirical results obtained by several European and North-American surveys. At first, few North-American small firms really use HR planning or job analysis. Some small businesses refer to short term recruitment objectives and prepare job descriptions, but they mostly comply with external obligations. However, this situation slightly differs in France and other countries where these practices are officially ruled or promoted. Secondly, a majority of small firms show little creativity or imagination in their staffing sources, sticking to a few passive or inefficient methods. The selection process is often restricted to the simple analysis of application forms and the use of personal interviews; selection criterias and tests are widely unused; and induction is typically limited to new employees being introduced to work place and peers, except for some participative settings. Finally, an analysis of the formalization level of employment and placement activities emphasizes the renewal of HRM approaches by means of discussing the eventual impacts of new management strategies on most organizations, and more specifically on small and medium-sized businesses.

\section{RESUMEN}

Este artículo resume un trabajo exploratorio orientado a identificar las principales prácticas de captación de recursos humanos en el contexto de las PyME. Para ello fué analizada la experiencia internacional a través de la documentación empírica que aporta resultados sobre el terreno en diversos paises de Europa y América del Norte. Una primera constatación revela que muy pocas empresas norteamericanas efectúan una planificación real de recursos humanos o un verdadero análisis de cargos. Algunas establecen un plan de reclutamiento a corto plazo y redactan una descripción de cargos, pero estas herramientas sólo sirven para cumplir reglamentaciones externas. La situación difiere ligeramente en Francia y en paises donde estas prácticas estan reglamentadas o promovidas. De otro lado, la mayoría de las PyME demuestran poca imaginación en la selección de fuentes de reclutamiento, limitándose a algunos métodos pasivos. La selección se limita al análisis de 
formularios de solicitud de empleo y a las entrevistas individuales. Los criterios de selección y los test aparecen fuertemente sub-utilizados y el recibimiento no pasa de la presentación a los colegas mas immediatos, con la excepción de PyME más participativas. En fin, el análisis del grado de formalización de prácticas de adquisición de Recursos Humanos hace ver la necesidad de reorientar el estudio de la Gestión de Recursos Humanos hacia la discusión sobre las implicaciones eventuales de una "GESTION RENOVADA" en las organizaciones, particularmente en las PyME.

\section{Introduction}

Les bouleversements économiques, politiques et sociaux des dernières décennies ont eu des effets considérables sur les ressources humaines (RH). Combinés aux phénomènes occidentaux de féminisation, de hausse du niveau de scolarité et de vieillissement de la main-d'œuvre, ils ont ouvert la porte à de nouvelles aspirations des travailleurs, notamment en matière de considération, d'autonomie, de communication, d'information et de participation (Fabi, 1991). L'intensification de ces préoccupations a rendu essentielle l'intégration de la gestion des ressources humaines (GRH) au processus stratégique des organisations. Dans un contexte international exigeant une main-d'œuvre qualifiée et correctement rémunérée, les entreprises n'ont d'autre choix que de poursuivre des objectifs dynamiques de mobilisation et de revitalisation de leur personnel. Même en petite et moyenne entreprise (PME), la GRH ne peut être considérée comme une simple annexe marginale aux autres domaines de la gestion, bien qu'elle donne lieu le plus souvent à des jugements globaux, fondés sur des impressions et des observations partielles plutôt que sur des analyses approfondies (Mahé de Boislandelle, 1988; Garand, 1992).

En réalité, toutes les facettes d'une organisation sont influencées par la fonction RH. Cette situation s'observe aisément par la place occupée dans la presse populaire par les PME ainsi que l'importance des problèmes de gestion du personnel parmi les causes de succès ou d'échec des PME. Bien que la majorité des propriétaires-dirigeants reconnaissent la nécessité fondamentale et l'importance de leurs employés, de nombreuses études démontrent qu'ils éprouvent des difficultés persistantes à gérer convenablement leur personnel (d'Amboise et Gasse, 1982; Mahé de Boislandelle et al., 1985). Selon ces enquêtes, les difficultés de recrutement se placeraient parmi les trois plus importants problèmes de gestion des PME (d'Amboise et Parent, 1989). Comme les entrepreneurs doivent souvent assumer eux-mêmes les responsabilités de GRH, il semble plausible de croire qu'un besoin pressant d'aide est attendu à ce niveau. Cependant, ces préoccupations n'ont donné lieu qu'à cinq recherches majeures (Baker, 1955 ; McEvoy, 1984 ; Mahé de Boislandelle, 1988 ; Hornsby 
et Kuratko, 1990; Garand, 1993) qui forment les principaux points d'ancrage de l'analyse des pratiques de GRH en PME'.

Les informations colligées dans le présent article contribuent directement à favoriser la diffusion de ces travaux empiriques appliqués aux PME. Maintenant que les appuis théoriques de la GRH sont établis en contexte de $\mathrm{PME}^{2}$, il apparaît essentiel de repousser les frontières traditionnelles de la GRH jusqu'aux véritables pratiques observées dans l'immense réservoir économique constitué par les PME.

Ainsi, dans la première partie, nous soumettons quelques considérations méthodologiques relatives à l'étude de la GRH dans les PME; nous présentons succinctement les études empiriques retenues et soulignons certains biais relevés dans les méthodologies. Dans la seconde partie de l'article, nous présentons un état des connaissances des pratiques d'acquisition des RH en PME; nous y décrivons les activités de planification des ressources humaines, d'analyse des emplois, de recrutement, de sélection et d'accueil, telles qu'observées sur le terrain par plus d'une quarantaine d'études empiriques.

\section{Considérations méthodologiques}

Basée sur une approche exploratoire, cette étude présente également une dimension non empirique impliquant l'utilisation d'outils de recherche documentaire, d'analyse thématique et d'analyse de contenu. Une recension systématique de la documentation en langues française et anglaise a permis de consulter et d'analyser plus d'une centaine de périodiques, collections et cahiers de recherche provenant d'Amérique du Nord et d'Europe de façon à repérer les articles et travaux portant sur une ou plusieurs pratiques de GRH en PME (Garand, 1993). Cette démarche s'est faite à l'aide des critères de sélection suivants :

1. La diffusion de ces travaux demeure restreinte et la plupart des études portant sur la GRH en PME s'avèrent peu accessibles dans leur version «technique ». C'est pourquoi notre rapport de recherche (Garand, 1993) est diffusé depuis mars 1993, tout comme le sera bientôt un ouvrage collectif sur la gestion des PME (GRH : chapitre 10) chez Economica (Paris).

2. Le lecteur aurait avantage à se référer à nos communications aux $2^{\mathrm{e}}, 3^{\mathrm{e}}$ et $4^{\mathrm{e}}$ Congrès de l'AGRH (Garand, 1992 ; Garand et Fabi, 1991, 1993 ; Fabi, Garand et Pettersen, 1993) pour mieux comprendre les concepts et fondements théoriques de la GRH en PME, ainsi qu'au dossier «État de la recherche » paru à l'automne 1992 dans la Revue Organisation (Garand et Fabi, 1992). 
1. études publiées entre 1950 et 1993 en milieu scolaire, professionnel et gouvernemental;

2. contenant des données empiriques explicites et originales traitant d'une ou plusieurs pratiques de GRH;

3. à partir d'un échantillon totalement ou partiellement constitué de PME (taille variant selon les pays: généralement inférieure à $500 \mathrm{em}-$ ployés $)^{3}$;

4. faisant l'analyse d'au moins un facteur de contingence influençant le degré de formalisation de la GRH en PME.

L'application de ces critères a facilité la sélection des études, permettant d'inclure certains travaux publiés dans des périodiques professionnels, en excluant toutefois une masse considérable d'articles diffusés dans des revues et magazines populaires, ces derniers ne contenant généralement que des résultats anecdotiques ou normatifs, sans mention de la méthodologie ou de la source des concepts et des références utilisés. Dans ce processus, 471 articles et documents de toutes catégories et provenances ont été considérés initialement, les volumes et traités en GRH et gestion de PME étant exclus de ce nombre puisqu'ils contiennent rarement des résultats empiriques explicites. La documentation pertinente a été résumée et ses résultats compilés, portés en tableaux et interprétés en prenant bien soin d'effectuer un découpage du contenu en unités significatives. À partir de ces données, classées par thèmes et par pratiques, l'analyse et l'interprétation s'est effectuée sur la base du cadre conceptuel élaboré auparavant (Garand, 1993). L'analyse des pratiques d'acquisition des RH en PME porte spécifiquement sur 42 études empiriques regroupant plus de 6000 entreprises de petite et moyenne dimensions.

3. En matière de GRH, les PME peuvent être classées selon la définition opérationnelle suivante (Garand, 1993) :

$\begin{array}{llr}\text { TPE } & \text { très petite entreprise } & \text { de } 0 \text { à } 19 \text { employés } \\ \text { PE } & \text { petite entreprise } & \text { de } 20 \text { à } 99 \text { employés } \\ \text { ME } & \text { moyenne entreprise } & \text { de } 100 \text { à } 249 \text { employés } \\ \text { MGE } & \text { moyenne grande entreprise } & \text { de } 250 \text { à } 499 \text { employés } \\ \text { GE } & \text { grande entreprise } & \text { de } 500 \text { à } 999 \text { employés } \\ \text { TGE } & \text { très grande entreprise } & \text { plus de } 1000 \text { employés }\end{array}$


TABLEAU 1

Compilation globale des études analysées : acquisition des ressources humaines

(fréquence et pourcentage du nombre total d'études, pour chaque rubrique)

\begin{tabular}{|c|c|c|c|c|c|c|c|c|c|c|c|c|c|c|}
\hline & \multicolumn{2}{|c|}{ why. } & \multicolumn{2}{|c|}{ xy. } & \multicolumn{2}{|c|}{ \%1\%11 } & \multicolumn{2}{|c|}{ 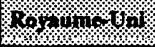 } & \multicolumn{2}{|c|}{ \% } & \multicolumn{2}{|c|}{ 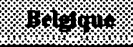 } & \multicolumn{2}{|c|}{ Wom } \\
\hline & $n$ & $\boldsymbol{x}$ & $n$ & $\%$ & $n$ & $\%$ & $n$ & $\%$ & $n$ & $\%$ & $n$ & $\%$ & $n$ & $\%$ \\
\hline Nombre of tudes retenves & 13 & 31,0 & 13 & 31,0 & 9 & 21,4 & 4 & 9,5 & 2 & 4,8 & 1 & 2,4 & 42 & 100,0 \\
\hline $\begin{array}{c}\text { Période drenquete: } \\
.1950-60 \\
.1900-60 \\
.1970-79 \\
.1900-84 \\
.1986-80 \\
.1000 . .\end{array}$ & $\begin{array}{l}1 \\
1 \\
2 \\
5 \\
4 \\
0\end{array}$ & $\begin{array}{r}7,7 \\
7,7 \\
15,4 \\
38,5 \\
30,8\end{array}$ & $\begin{array}{l}0 \\
0 \\
1 \\
2 \\
9 \\
1\end{array}$ & $\begin{array}{r}7,7 \\
15,4 \\
69,2 \\
7,7\end{array}$ & $\begin{array}{l}0 \\
0 \\
1 \\
6 \\
1 \\
1\end{array}$ & $\begin{array}{l}11,1 \\
66,7 \\
11,1 \\
11,1\end{array}$ & $\begin{array}{l}0 \\
0 \\
0 \\
1 \\
2 \\
1\end{array}$ & $\begin{array}{l}25,0 \\
50,0 \\
25,0\end{array}$ & $\begin{array}{l}0 \\
0 \\
1 \\
0 \\
1 \\
0\end{array}$ & $\begin{array}{l}50,0 \\
50,0\end{array}$ & $\begin{array}{l}0 \\
0 \\
0 \\
1 \\
0 \\
0\end{array}$ & 100,0 & $\begin{array}{r}1 \\
1 \\
5 \\
15 \\
17 \\
3\end{array}$ & $\begin{array}{r}2,4 \\
2,4 \\
11,9 \\
35,7 \\
40,5 \\
7,1\end{array}$ \\
\hline $\begin{array}{l}\text { Statut des auteurs } t \text { : } \\
\text { - profeceour universitaire } \\
\text { - ctudiant } 2^{\circ} \text { ou } 3^{\circ} \text { cycle } \\
\text { - profecelonnel - concultant }\end{array}$ & $\begin{array}{r}21 \\
0 \\
0\end{array}$ & 100,0 & $\begin{array}{r}10 \\
3 \\
3\end{array}$ & $\begin{array}{r}62,5 \\
18,8 \\
18,8\end{array}$ & $\begin{array}{l}8 \\
3 \\
2\end{array}$ & $\begin{array}{l}61,5 \\
23,1 \\
15,4\end{array}$ & $\begin{array}{l}0 \\
0 \\
0\end{array}$ & 100,0 & $\begin{array}{l}4 \\
0 \\
0\end{array}$ & 100,0 & $\begin{array}{l}2 \\
0 \\
0\end{array}$ & 100,0 & $\begin{array}{r}54 \\
6 \\
5\end{array}$ & $\begin{array}{r}83,1 \\
9,2 \\
7,7\end{array}$ \\
\hline 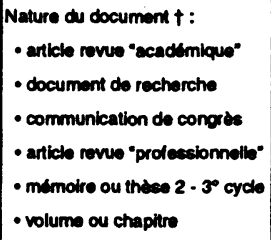 & $\begin{array}{l}9 \\
0 \\
5 \\
2 \\
1 \\
1\end{array}$ & $\begin{array}{r}50,0 \\
27,8 \\
11,1 \\
5,6 \\
5,6\end{array}$ & $\begin{array}{l}6 \\
7 \\
2 \\
8 \\
2 \\
2\end{array}$ & $\begin{array}{r}22,2 \\
25,9 \\
7,4 \\
29,6 \\
7,4 \\
7,4\end{array}$ & $\begin{array}{l}2 \\
6 \\
2 \\
0 \\
2 \\
2\end{array}$ & $\begin{array}{r}14,3 \\
42,9 \\
14,3 \\
14,3 \\
14,3\end{array}$ & $\begin{array}{l}1 \\
1 \\
1 \\
0 \\
1 \\
0\end{array}$ & $\begin{array}{l}25,0 \\
25,0 \\
25,0 \\
25,0\end{array}$ & $\begin{array}{l}1 \\
1 \\
1 \\
0 \\
0 \\
0\end{array}$ & $\begin{array}{l}33,3 \\
33,3 \\
33,3\end{array}$ & $\begin{array}{l}0 \\
1 \\
0 \\
0 \\
0 \\
0\end{array}$ & 100,0 & $\begin{array}{r}19 \\
16 \\
11 \\
10 \\
6 \\
5\end{array}$ & $\begin{array}{r}28,4 \\
23,9 \\
16,4 \\
14,9 \\
9,0 \\
7,5\end{array}$ \\
\hline $\begin{array}{l}\text { Taillo des dchantillons utilises } t \text { : } \\
\text { - molns de } 20 \text { PME } \\
\text { - de } 20 \text { a } 49 \\
\text { - de } 50 \text { a } 99 \\
\text { - de } 100 \text { a } 399 \\
\text { - de } 400 \text { a } 999\end{array}$ & $\begin{array}{l}1 \\
2 \\
5 \\
4 \\
1\end{array}$ & $\begin{array}{r}7,7 \\
15,4 \\
38,5 \\
30,8 \\
7,7\end{array}$ & $\begin{array}{l}3 \\
4 \\
1 \\
4 \\
1\end{array}$ & $\begin{array}{r}23,1 \\
30,8 \\
7,7 \\
30,8 \\
7,7\end{array}$ & $\begin{array}{l}1 \\
2 \\
4 \\
1 \\
0\end{array}$ & $\begin{array}{l}12,5 \\
25,0 \\
50,0 \\
12,5\end{array}$ & $\begin{array}{l}1 \\
1 \\
0 \\
2 \\
0\end{array}$ & $\begin{array}{l}25,0 \\
25,0 \\
50,0\end{array}$ & $\begin{array}{l}0 \\
0 \\
1 \\
1 \\
0\end{array}$ & $\begin{array}{l}50,0 \\
50,0\end{array}$ & $\begin{array}{l}0 \\
1 \\
0 \\
0 \\
0\end{array}$ & 100,0 & $\begin{array}{r}6 \\
10 \\
11 \\
12 \\
2\end{array}$ & $\begin{array}{r}14,6 \\
24,4 \\
26,8 \\
29,3 \\
4,9\end{array}$ \\
\hline
\end{tabular}

t : le nombre total des " $n$ " peut différer du nombre détudes retenues pour chaque pays correspondant 
Ce mouvement d'intérêt envers les PME transparaît de façon limpide au tableau 1, qui regroupe certaines données descriptives des 42 études empiriques portant sur l'acquisition des RH en PME. On y note la concentration des enquêtes depuis 1980 (83\% pour l'ensemble des pays), le statut professoral des chercheurs $(83 \%)$, et la disparité des médias utilisés pour la diffusion de ces recherches. Il convient aussi de préciser la provenance internationale des travaux, dont l'importante proportion d'études menées aux États-Unis et en France, ces deux pays regroupant à eux seuls $62 \%$ des travaux retenus. Avant d'aborder l'analyse des pratiques réellement appliquées en PME, il faut bien comprendre les contraintes imposées par l'importante hétérogénéité des travaux réalisés dans ce domaine : périodes d'enquête, populations diversifiées, échantillons variés et parfois trop étendus, méthodologies différentes, interprétations plus ou moins développées, publications professionnelles ou académiques, travaux universitaires ou étudiants, etc. Tous ces facteurs compliquent singulièrement la description et l'analyse des pratiques de GRH en PME, rendant parfois impossible toute comparaison des résultats bruts.

\subsection{Analyse méthodologique des études empiriques}

L'analyse méthodologique des études empiriques traitant d'acquisition des RH en PME corrobore substantiellement le préjugé observé à maintes reprises lors de congrès internationaux réunissant des chercheurs européens et nordaméricains: les méthodes d'enquête et l'utilisation des études empiriques en GRH diffèrent largement d'un continent à l'autre. Ainsi, l'enquête sur le terrain détient une importance capitale en Amérique du Nord, comparativement à l'Europe où les travaux théoriques et conceptuels en GRH reçoivent davantage d'attention et souffrent parfois d'un manque de données empiriques fondamentales (tableau 2).

Primo, 90\% des études utilisent un questionnaire d'enquête, les proportions variant surtout en fonction des pays, mais aussi du recours aux études de cas, aux monographies d'entreprises et aux bases de données informatisées, lesquelles occupent cependant une position très secondaire.

Secundo, seulement $32 \%$ des travaux analysés mentionnent explicitement l'utilisation d'un prétest avant de passer à l'enquête proprement dite. S'agit-il d'un oubli dans la description de leur méthodologie ou d'une lacune expérimentale majeure ? En outre, peu d'études empiriques (26\%) effectuent un suivi après l'enquête, soit pour compléter les questionnaires inachevés, soit pour valider ou contrôler le processus d'enquête. Cependant, ces observations ne constituent que des indices bien relatifs des tendances réelles, car la plupart des études retenues ne fournissent pas toutes les informations relatives à leur méthodologie. 
TABLEAU 2

Aperçu global des méthodologies d'enquête utilisées : acquisition des ressources humaines

(fréquence et pourcentage; pour chaque rubrique; par pays)

\begin{tabular}{|c|c|c|c|c|c|c|c|c|c|c|c|c|c|c|}
\hline & \multicolumn{2}{|c|}{ mon. } & \multicolumn{2}{|c|}{4} & \multicolumn{2}{|c|}{ 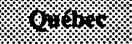 } & \multicolumn{2}{|c|}{ 6\% } & \multicolumn{2}{|c|}{ s.r. } & \multicolumn{2}{|c|}{ \%. } & \multicolumn{2}{|c|}{ 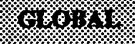 } \\
\hline & $n$ & $x$ & $n$ & $x$ & $n$ & $x$ & $n$ & $\%$ & $n$ & $x$ & $n$ & $\%$ & $n$ & $\%$ \\
\hline (n) & \% & & \% & & 8 & & 幽 & & 梦 & & 率 & & 格 & \\
\hline Utilleation ofun questionnaire $\uparrow$ & 11 & 84,6 & 13 & 100,0 & 8 & 88,9 & 3 & 75,0 & 2 & 100,0 & 1 & 100,0 & 38 & 90,5 \\
\hline $\begin{array}{l}\text { Administration du questionnaire } \\
\text { - avec protect } \\
\text { - envol postal } \\
\text { - aucune entrevie } \\
\text { - entrevice en personne } \\
\text { - suivi post-entrevie }\end{array}$ & $\begin{array}{l}3 \\
7 \\
7 \\
4 \\
4\end{array}$ & $\begin{array}{l}27,3 \\
63,6 \\
63,6 \\
36,4 \\
36,4 \\
\end{array}$ & $\begin{array}{l}5 \\
4 \\
4 \\
9 \\
4\end{array}$ & $\begin{array}{l}38,5 \\
30,8 \\
30,8 \\
69,2 \\
30,8 \\
\end{array}$ & $\begin{array}{l}3 \\
5 \\
4 \\
4 \\
2\end{array}$ & $\begin{array}{l}37,5 \\
62,5 \\
50,0 \\
50,0 \\
25,0\end{array}$ & $\begin{array}{l}1 \\
1 \\
1 \\
2 \\
0\end{array}$ & $\begin{array}{l}33,3 \\
33,3 \\
33,3 \\
66,7\end{array}$ & $\begin{array}{l}0 \\
2 \\
2 \\
0 \\
0\end{array}$ & $\begin{array}{l}100,0 \\
100,0\end{array}$ & $\begin{array}{l}0 \\
0 \\
0 \\
1 \\
0\end{array}$ & 100,0 & $\begin{array}{l}12 \\
19 \\
18 \\
20 \\
10\end{array}$ & $\begin{array}{l}31,6 \\
50,0 \\
47,4 \\
52,6 \\
26,3\end{array}$ \\
\hline $\begin{array}{l}\text { Autres modes denquele } t \text { : } \\
\text { - panel } \\
\text { - Etude de cas, monographio } \\
\text { - bace de donndes } \\
\text { - autre }\end{array}$ & $\begin{array}{l}1 \\
0 \\
0 \\
1\end{array}$ & $\begin{array}{l}7,7 \\
7,7\end{array}$ & $\begin{array}{l}0 \\
1 \\
0 \\
0\end{array}$ & 7,7 & $\begin{array}{l}0 \\
0 \\
1 \\
0\end{array}$ & 11,1 & $\begin{array}{l}0 \\
1 \\
0 \\
0\end{array}$ & 25,0 & $\begin{array}{l}0 \\
0 \\
0 \\
0\end{array}$ & & $\begin{array}{l}0 \\
0 \\
0 \\
0\end{array}$ & & $\begin{array}{l}1 \\
2 \\
1 \\
1\end{array}$ & $\begin{array}{l}2,4 \\
4,8 \\
2,4 \\
2,4\end{array}$ \\
\hline Etudes longltudinales t & 1 & 7,7 & 1 & 7,7 & 0 & & 1 & 25,0 & 0 & & 0 & & 3 & 7,1 \\
\hline $\begin{array}{l}\text { Statut des intenvieweurs }{ }^{\circ}: \\
\text { - prolesceurs universitaires } \\
\text { - Átudlants universitaires } \\
\text { - protessionnels } \\
\end{array}$ & $\begin{array}{l}1 \\
2 \\
1\end{array}$ & • & $\begin{array}{l}5 \\
5 \\
3\end{array}$ & • & $\begin{array}{l}3 \\
1 \\
1\end{array}$ & • & $\begin{array}{l}2 \\
0 \\
1\end{array}$ & - & $\begin{array}{l}0 \\
0 \\
0\end{array}$ & & $\begin{array}{l}1 \\
0 \\
0\end{array}$ & $\bullet$ & $\begin{array}{r}12 \\
8 \\
6\end{array}$ & $\begin{array}{l}46,2 \\
30,8 \\
23,1\end{array}$ \\
\hline $\begin{array}{l}\text { Enqueles mentes auprès de }{ }^{\circ} \text { : } \\
\text { - proprietaires-dirigeants } \\
\text { - responsables du personnel } \\
\text { - cactes superleurs } \\
\text { - employes }\end{array}$ & $\begin{array}{l}7 \\
4 \\
4 \\
2\end{array}$ & $\begin{array}{l}41,2 \\
23,5 \\
23,5 \\
11,8\end{array}$ & $\begin{array}{l}11 \\
8 \\
8 \\
2\end{array}$ & $\begin{array}{r}37,9 \\
27,6 \\
27,6 \\
6,9\end{array}$ & $\begin{array}{l}4 \\
6 \\
4 \\
0\end{array}$ & $\begin{array}{l}28,6 \\
42,9 \\
28,6\end{array}$ & $\begin{array}{l}4 \\
0 \\
1 \\
2\end{array}$ & $\begin{array}{l}57,1 \\
14,3 \\
28,6\end{array}$ & $\begin{array}{l}0 \\
2 \\
0 \\
0\end{array}$ & 100,0 & $\begin{array}{l}1 \\
0 \\
0 \\
0\end{array}$ & 100,0 & $\begin{array}{r}27 \\
20 \\
17 \\
6\end{array}$ & $\begin{array}{r}38,6 \\
28,6 \\
24,3 \\
8,6\end{array}$ \\
\hline
\end{tabular}

N.B. : les études empiriques retenues ne fournissent pas toutes les informations relatives à leur méthodologie denquete

$t$ : en fonction du nombre total dóftudes retenues pour chaque pays ( $\left(1^{\circ}\right.$ ligne)

- : selon le total des réponses à cette rubrique, par pays

- : en fonction du nombre total dótudes utilisant un questionnaire, par pays $\left(2^{\circ}\right.$ ligne) 
Tertio, on note une différence marquée dans les modalités d'administration des questionnaires entre l'Europe et l'Amérique du Nord. En effet, les chercheurs européens se servent très peu $(29 \%)$ des enquêtes postales (sans entrevue) et recourent majoritairement aux entrevues directes (71\%), tandis que les Nord-Américains utilisent abondamment les envois postaux (81\%) sans effectuer d'entrevue, seulement $38 \%$ des questionnaires y étant complétés lors d'entrevues en personne. Incidemment, il faut souligner que les études québécoises recourent dans $50 \%$ des cas $(n=4)$ aux entrevues directes, comparativement à $36 \%$ aux États-Unis $(\mathrm{n}=4)$, ce qui représente peut-être une indication du caractère biculturel du Québec.

Toutefois, les enquêtes par questionnaires retournés par la poste s'avèrent très peu appropriées au contexte des PME. On peut même considérer ce mode d'enquête comme déficient en PME, car une forte proportion des questionnaires retournés par voie postale ont souvent été complétés par un cadre ou un employé administratif, au lieu du propriétaire-dirigeant qui détient habituellement la véritable réponse aux questions posées. Bien que cette procédure constitue une pratique courante en GE, elle apparaît fortement discutable en PME. Dans la plupart des cas, il serait préférable de recourir aux entrevues directes afin de s'assurer de la participation concrète de l'entrepreneur et d'éviter d'importants biais méthodologiques liés aux répondants.

Les interviewers sont majoritairement des professeurs d'université (46\%). Les enquêtes portant sur l'acquisition des RH en PME s'adressent principalement aux propriétaires-dirigeants (39\%) et aux responsables des RH (29\%), bien que les autres catégories de personnel occupent une place importante (33\%). Il semble aussi pertinent de signaler l'absence complète d'entrevues téléphoniques dans ces études empiriques, le recours au téléphone étant limité aux premières approches avec l'entreprise, à la sélection des participants, à la prise de rendezvous, au rappel des questionnaires et à la correction des réponses mal complétées par les répondants ou les enquêteurs. En outre, on ne retrouve que trois études longitudinales, et deux d'entre elles ne s'étendent que sur une période d'un an. Par contre, il faut souligner la persistance du Panel des PMI Lorraines (France) qui poursuit depuis 1988 ses analyses auprès de 400 PME industrielles (Bayad, 1991; 1992).

Finalement, l'analyse méthodologique des études retenues a fait ressortir plusieurs faiblesses, liées pour la plupart aux impondérables de la recherche empirique en contexte de PME. En premier lieu, les schèmes expérimentaux demeurent généralement peu complexes et souvent inexistants : les objectifs de recherche paraissent limités à une description ponctuelle, sans tenter d'établir de liens de corrélation ou d'autres relations entre quelques variables présentées formellement. En deuxième lieu, la taille et la répartition des échantillons par 
catégorie de taille laisse voir de nombreuses lacunes : échantillons réduits, établis sans véritable rigueur méthodologique, trop localisés et répartis de façon aléatoire entre les catégories de taille. En troisième lieu, les études empiriques traitant d'acquisition des RH en PME couvrent trop souvent plusieurs secteurs d'activité non comparables, caractérisés par des besoins de main-d'œuvre, des marchés et des contraintes matérielles fortement différenciés. Cette hétérogénéité des échantillons soulève à nouveau des questionnements relatifs à la fidélité et à la validité des résultats, d'autant plus que notre analyse tente de les comparer entre eux. En quatrième lieu, il importe de rappeler le caractère inapproprié en PME de l'enquête par questionnaires retournés par voie postale. En cinquième lieu, il apparaît très difficile, voire le plus souvent impossible, d'avoir recours à des méthodes d'analyse statistique sophistiquées lorsqu'on traite des données provenant d'échantillons de PME. En réalité, on doit généralement se limiter à dégager des tendances lourdes basées principalement sur des statistiques descriptives et sur des tests de comparaison.

\subsection{Critique des échantillons de certaines études}

Quelques études ont exigé une attention particulière à la suite de l'observation de biais dans les échantillons ou les méthodes de recherche. Bien que chaque étude empirique puisse laisser place à une saine critique méthodologique, nous ne commentons que les biais les plus flagrants, afin de modérer l'interprétation des résultats empiriques présentés à la section deux.

1. L'importante étude de Baker (1955) paraît aujourd'hui quelque peu vétuste, puisque ses résultats, tirés de PME de l'après-guerre, proviennent d'une enquête réalisée en 1950 dans des entreprises manufacturières et industrielles de l'Ohio, à une époque où les préoccupations relatives à la GRH moderne et aux PME étaient à leurs balbutiements. Cependant, sa méthodologie s'appuie sur des normes scientifiques encore valables dans les décennies 80 et 90 .

2. Mealiea et Lee (1980) ont réalisé une étude basée sur la propriété des entreprises, et leur échantillon se compose de firmes canadiennes et américaines qui peuvent être des sociétés-mères, des filiales, des établissements ou des entreprises autonomes. Toutefois, bien que les résultats soient désagrégés en fonction du critère de propriété, il s'avère fort réaliste de croire qu'une proportion substantielle d'entreprises comptant moins de 499 employés soient des établissements dépendants d'organisations de plus grande taille, faussant ainsi les tendances observées en ce qui concerne la participation décisionnelle du SRH aux questions de GRH. 
3. Paumier et Gouadain (1984), dont les travaux concernent principalement la planification des ressources humaines, ont fixé leur plancher à 50 salariés, et leurs catégories de taille organisationnelle semblent beaucoup trop étendues, regroupant des entreprises de 70 à 299 salariés, de 200 à 749, et de plus de 750 individus. En outre, leur enquête apparaît quelque peu biaisée par la formulation même de leur approche, fortement axée sur les prévisions économiques et sociales au détriment des activités concrètes de planification des ressources humaines.

4. Mahé de Boislandelle $(1985,1988,1990,1993)$ rapporte une masse considérable d'information pertinente aux pratiques de GRH en PME, mais il ne fournit pas explicitement de détails relatifs aux échantillons, aux méthodes de collecte et d'analyse des données, à sa méthodologie globale d'enquête et aux caractéristiques sociodémographiques des répondants (Fabi et Garand, 1989), en plus de présenter des résultats peu appuyés par des données de terrain. Cependant, après des vérifications auprès de l'auteur, nous avons constaté que la majorité de ses résultats provenaient de travaux effectués par des étudiants universitaires dans le cadre de travaux dirigés, sous sa direction et à l'aide d'une méthodologie constante, ce qui semble être une pratique assez répandue en France (tableau 2).

5. Lépine (1986) présente des résultats empiriques difficiles à interpréter au sujet des sources internes et externes de recrutement du personnel, sans désagrégation systématique en fonction de la taille organisationnelle. De plus, ses données regroupent dans des ensembles inhabituels les diverses méthodes de recrutement, compliquant quelque peu l'analyse des impacts réels de chaque technique.

6. Thacker et Cattaneo (1987) ont eu recours à des catégories trop étendues de taille organisationnelle, soit de 0 à 200 employés, 201 à 1000 et 1000 et plus. En outre, leur questionnaire était adressé aux directeurs du personnel, laissant soupçonner que plusieurs PME, qui ne disposaient d'aucun responsable des ressources humaines, n'ont tout simplement pas participé à l'enquête, effectuée par voie postale. Cette situation semble d'ailleurs constituer une carence méthodologique assez répandue dans les études en GRH et provoque des biais importants lorsqu'on utilise des PME sans s'adapter à leur contexte particulier.

7. Audet et al. (1987) abordent plusieurs pratiques de GRH selon une approche descriptive (études de cas) et utilisent un échantillon de 20 entreprises québécoises où 5 des $7 \mathrm{ME}$ visitées sont des GE ou 
des filiales directes de TGE, nommément Cascades, Kimberley-Clark, Textiles Dionne et Pétromont. En fait, les exceptions mentionnées dans l'étude représenteraient plutôt la situation réelle de la GRH en ME. Quant aux PE, elles occupent une position secondaire dans leur étude, parmi un ensemble de considérations relatives aux moyennes et grandes dimensions.

8. McEvoy $(1983,1984)$ souligne explicitement le biais provoqué par les répondants eux-mêmes, $50 \%$ d'entre eux étant seuls responsables des activités de gestion du personnel de leur établissement, tout en ne disposant pas des connaissances suffisantes en GRH pour être insatisfaits de leurs propres pratiques. Cet état de fait représenterait un des pièges les plus dangereux de l'étude des pratiques de GRH en contexte de PME. En effet, puisque l'enquêteur provient d'un milieu universitaire fortement imprégné de l'ensemble des connaissances scientifiques en GRH, son approche risque de dépasser largement le niveau cognitif des répondants, qui auront alors des difficultés à associer certaines de leurs pratiques usuelles aux termes et concepts spécialisés du questionnaire. Si, par surcroît, l'enquête est menée par voie postale, ces difficultés peuvent entraîner des biais considérables, limitant d'autant la validité des résultats. Incidemment, McEvoy (1983, 1984) demeure l'un des seuls chercheurs américains à avoir utilisé l'entrevue directe, avec Robinson (1981), Verser (1987), Hornsby et Kuratko (1990), comme le démontre le tableau 2.

9. Les enquêtes professionnelles de Le Louarn et Thériault (1984) comportent aussi certains biais. Les échantillons comprennent plusieurs filiales de compagnies américaines de taille probablement inférieure à 500 employés et les résultats associés aux PME seraient alors confondus de manière indissociable. L'observation des organisations participantes laisse d'ailleurs entrevoir une proportion de PME «réelles » estimée à 10-12\%, comparativement aux 23 et $28 \%$ de moins de 250 employés présentés par les auteurs. En effet, après une brève analyse qualitative de la liste des organisations participantes, nous retrouvons des hôpitaux, des cégeps, des municipalités et des GE aux noms fort connus qui sont qualifiés de PME selon leur nombre d'employés, sur une base d'établissement. Il va sans dire que de tels établissements peuvent être qualifiés de petites ou moyennes organisations quoique en matière de ressources humaines, on y retrouve des conditions très proches du système bureaucratique gouvernemental, largement différent du contexte propre aux PME du secteur privé. L'interprétation des résultats de ces enquêtes doit se faire avec quel- 
ques réserves, puisque $50 \%$ des organisations de petite taille (moins de 250 employés) ne peuvent y être qualifiées de PME au sens propre du terme.

10. Vermot-Gaud (1987) ne mentionne sur sa méthodologie que l'utilisation d'entrevues directes auprès de dirigeants et de directeurs du personnel de 30 entreprises françaises comptant entre 50 et 1000 salariés, et ne donne même pas les coordonnées exactes de son étude empirique, effectuée en 1983 sous le parrainage de la CEE.

Bien entendu, plusieurs autres études empiriques comportent des lacunes méthodologiques (échantillons ou autres), mais nous avons volontairement limité cette critique aux biais les plus manifestes, principalement pour les travaux qui fournissent une quantité importante de données. Néanmoins, tous ces résultats offrent des indications précieuses sur les tendances lourdes caractérisant les pratiques de GRH en PME et c'est pourquoi nous les avons retenus dans cette analyse. Ainsi, il importe de considérer globalement les difficultés posées par la recherche empirique en contexte de PME: le domaine n'a pas encore de fondements aussi rigoureux que d'autres disciplines des sciences humaines et sociales; les échantillons homogènes sont particulièrement difficiles à constituer; l'administration des questionnaires se fait plus difficilement qu'en GE; et enfin, les bases de comparaison demeurent fort rares et dispersées, compliquant davantage l'analyse comparative des données colligées et rendant parfois impossible l'utilisation de méthodes statistiques sophistiquées.

\section{Acquisition des ressources humaines en PME: état des connaissances}

Cette seconde partie s'inscrit dans une démarche globale visant à mieux faire connaître les particularités des pratiques de GRH en PME. Elle permet de saisir l'importance croissante des PME dans le renouveau industriel et socioéconomique de la majorité des pays industrialisés en présentant une analyse approfondie des activités traditionnellement associées à l'acquisition des RH. On y retrouve ainsi une synthèse des activités de planification des RH (organigrammes, décisions et planification stratégique), de l'analyse des emplois (description de tâches), du recrutement (décisions et responsabilités, sources et méthodes), de la sélection (décisions et responsabilités, techniques, références, tests, discrimination), de l'accueil, ainsi qu'un aperçu des difficultés et de l'efficacité du processus de recrutement et sélection (validation). Dès qu'il est question de croissance, les PME et leurs RH apparaissent dorénavant comme une préoccupation de premier plan, ouvrant bien grandes les frontières de la GRH. 


\subsection{Planification des ressources humaines}

Rarement perçus comme prioritaires, l'établissement de prévisions de maind'œuvre, de besoins de formation et l'élaboration d'organigrammes fonctionnels constituent des démarches formelles que peu de PME réussissent à concrétiser. Les politiques relatives à la planification des RH s'avèrent peu explicites, voire inexistantes, et bien que plusieurs propriétaires-dirigeants estiment faire des prévisions de RH, presque aucun n'a de document formalisé à cette fin. Ces activités semblent s'intégrer à la démarche globale de planification de l'entrepreneur qui demeure essentiellement intuitive, informelle et non verbalisée. Cependant, leur degré de formalisation s'accroît avec la taille, en parallèle à l'ensemble des politiques et procédures organisationnelles, et dans le cadre d'un processus décisionnel qui se développe peu à peu en véritable planification stratégique formelle en grande entreprise (GE). Deux tendances s'affrontent à ce sujet: les études qui relatent l'existence d'une planification des RH relativement articulée en PME et celles qui signalent l'absence de gestion prévisionnelle en PME.

D'une part, une majorité de PME régionales françaises déploient des efforts pour gérer prévisionnellement leurs RH (Paumier et Gouadain, 1984). Ces efforts ne restent pas superficiels, mais s'appuient sur des informations précises provenant des différents services concernés. La plupart des entreprises établissent des prévisions économiques et de mouvements de personnel pour un terme généralement inférieur à trois ans. Cependant, l'existence d'un plan de recrutement et d'un plan de mutations et promotions préétabli pour les cadres et non-cadres apparaît beaucoup plus rare en PME comme en GE. En effet, 70\% des PME planifient moins d'un mois à l'avance leur recrutement et $50 \%$ font de même pour les promotions (McEvoy, 1984).

Il existe bien une pratique de gestion de l'emploi en PME (tableau $3^{4}$ ). Elle serait toutefois plus réactive (contraintes structurelles) que préventive ou prévisionnelle (stratégie ou plan) et constituerait davantage un paramètre intégré ex-post au management de l'entreprise qu'un élément ex-ante, comme le serait la stratégie technologique. Cette gestion des effectifs dépendrait autant

4. Lecture des données statistiques présentées dans les tableaux. Les pourcentages indiquent que $\mathrm{XX} \%$ à $Y Y \%$ des PME échantillonnées (résultats tirés de diverses études empiriques) possèdent ou utilisent telle ou telle méthode $\mathrm{A}, \mathrm{B}$ ou $\mathrm{C}$, généralement appliquée dans cette pratique de GRH. Les flèches indiquent une proportion $(\%)$ croissante $(\boldsymbol{)})$ ou décroissante $(\boldsymbol{})$ en fonction de la taille organisationnelle (en nombre d'employés) ; la double flèche inversée $(\leftrightarrow)$ signifiant que les pourcentages ne diffèrent pas significativement selon la taille des entreprises. 
du stade de développement et de la maturité industrielle de la PME que de ses modes de production et de sa taille organisationnelle (Bayad et Herrmann, 1991). Elle se présente donc de façon contingente, cohérente avec les aspirations fondamentales du propriétaire-dirigeant, en osmose avec les orientations stratégiques de base de l'entreprise (avantage compétitif par les coûts ou par les services) compte tenu des contraintes et incertitudes de l'environnement (état de dépendance et de vulnérabilité de la firme dans son environnement concurrentiel). Cette gestion des effectifs se caractérise en PME par une grande variété de pratiques: souvent informelles, intuitives et peu planifiées, relevant directement de l'entrepreneur qui assume, entrevoit et exécute lui-même ou en collaboration les tâches de recrutement. On n'y retrouve pas de modèle unique de comportement, mais quelques tendances lourdes: recours croissant à l'externalisation de l'emploi, faiblesse relative des cas de substitution de ressources techniques aux RH, utilisation privilégiée du contrat à durée déterminée et heures supplémentaires comme ajustement à court terme (Naro, 1990).

D'autre part, Hess (1987) en arrive à des conclusions diamétralement opposées. Les PMI enquêtées ne gèrent pas préventivement leur personnel et l'analyse des besoins et ressources est presque toujours inexistante ou embryonnaire. Une majorité de répondants réalisent des prévisions de personnel, mais les modes d'adéquation ne relèvent pas d'une démarche planifiée (voir tableau 3). Il s'agit plutôt de décisions hâtives, sous pression, soit par manque de temps et de moyens ou par manque de compétences, soit parce que ces firmes considèrent que ces données ne sont utiles qu'en GE ou qu'elles estiment pouvoir évaluer leur personnel sans procédure formalisée. Les PME n'ont pas de plan de recrutement ou de promotion; aucune ne fait de prévision formalisée, ni n'a de document prospectif; il n'y a aucune prévision à court ou à long terme pour les ouvriers et employés. De plus, les PME éprouvent souvent des difficultés à percevoir les écarts entre ressources et besoins de personnel : la perception des besoins futurs s'appuie sur les expériences du passé, les perceptions personnelles de l'avenir de l'entreprise et $83 \%$ ne perçoivent pas d'écart entre leurs ressources et leurs besoins actuels.

Par ailleurs, ces questions n'ont reçu qu'une attention limitée en Amérique du Nord et il y aurait des faiblesses marquées dans ce domaine. La planification des besoins en RH semble souvent irréalisable ou inutile au dirigeant de PME : plusieurs entreprises produisant sur demande ou fonctionnant par soumission pour l'obtention de contrats font face à des fluctuations importantes d'activités. Il arrive aussi que les dirigeants ne disposent que de quelques heures pour combler un poste (Larrivée et d'Amboise, 1989), ce qui restreint singulièrement les possibilités d'établir des prévisions. 
TABlEAU 3

Planification des ressources humaines en PME

(grandes tendances observées; données tirées de plusieurs études empiriques; par ordre décroissant d'importance)

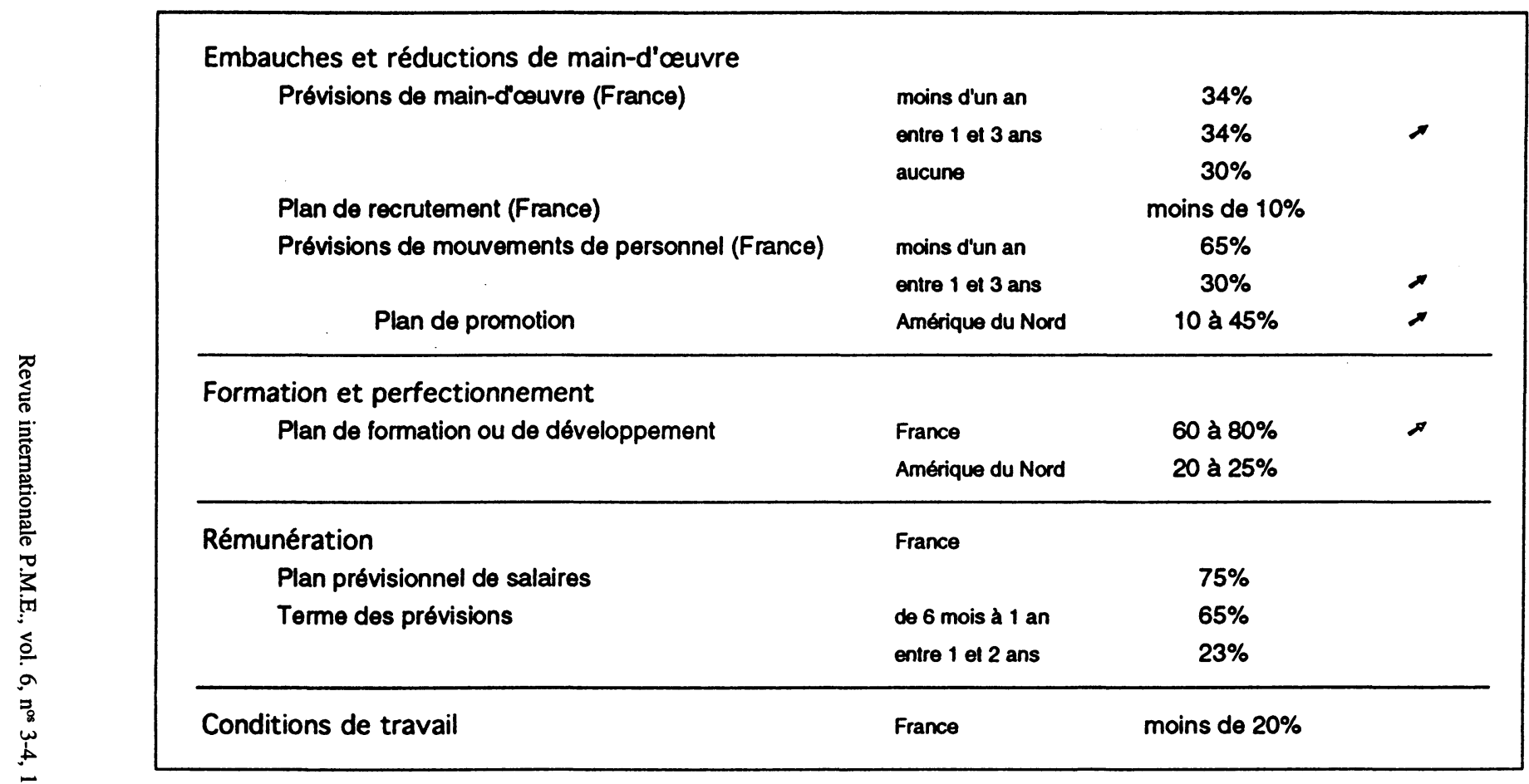




\subsubsection{Organigrammes}

L'organigramme constitue l'un des outils fondamentaux d'une certaine planification des RH en PME, bien qu'il n'y soit pas toujours formellement intégré. Entre $30 \%$ et $40 \%$ des PME possèdent un organigramme fonctionnel de l'entreprise (tableau 4) et ce pourcentage semble directement relié à la taille (AmbaRao et Pendse, 1982; Mahé de Boislandelle et al., 1985; Venet, 1988; Benoit et Rousseau, 1990). La structure organisationnelle des PE étant relativement simple et informelle, on présume qu'elle est bien comprise des employés, éliminant de ce fait le recours à un tableau formel qui déterminerait les statuts et liens d'autorité fonctionnels dans l'entreprise.

\section{TABleau 4}

\section{Utilisation de l'organigramme fonctionnel}

\begin{tabular}{lrr}
\hline en TPE & $(0-19$ employés $)$ & moins de $20 \%$ \\
en PE & $(20-99$ employés $)$ & 25 à $35 \%$ \\
en ME & $(100-249$ employés $)$ & 45 à $60 \%$ \\
en MGE & $(250$ à 500 employés $)$ & 70 à $80 \%$ \\
en GE & $(500$ employés et plus $)$ & plus de $75 \%$ \\
\hline
\end{tabular}

\subsubsection{Décisions et planification stratégique}

On retrouve aussi en PME certains éléments relatifs à la prise de décision et à l'élaboration de la planification stratégique de l'entreprise. Plus l'organisation est grande, plus on observe de niveaux hiérarchiques, ce qui signifie pour les responsables du personnel qu'il est souvent beaucoup plus long d'approuver, de modifier ou de mettre en application des décisions, politiques ou procédures; qu'il faut souvent d'innombrables signatures pour régler un problème anodin; que les employés au bas de l'échelle du service des RH hésitent souvent à faire valoir leur opinion à leur supérieur, même s'ils œuvrent eux aussi en RH. Dans un environnement hiérarchisé, le preneur ultime des décisions en RH est rarement formé en GRH. Il s'agit plutôt d'un cadre exécutif dont les responsabilités incluent les RH et qui s'appuie sur l'expertise de subalternes. En matière de participation des responsables du personnel à la prise de décision, ceux-ci participent à $76 \%$ au comité de direction et à $54,5 \%$ au comité de planification des opérations. Au sein de ces comités, plus de $50 \%$ des directeurs du personnel sont consultés sur les objectifs et projets de la firme pour l'introduction de 
nouvelles technologies ou de nouveaux produits (20 à $40 \%)$, et lors de la formulation des principaux objectifs dans les domaines liés aux RH. Toutefois, ils sont moins souvent consultés pour des objectifs touchant d'autres sphères d'activités, mais à $90 \%$ dans l'établissement de la politique de négociation collective et au moment d'une proposition de règlement (Gosselin, 1979).

\subsection{Analyse des emplois}

L'analyse des emplois n'a jamais été considérée comme une priorité en PME et, dans bien des cas, le propriétaire-dirigeant n'a aucune connaissance de cette pratique de GRH. En fait, le seul élément qu'on y retrouve demeure la description de tâches, élaborée principalement à partir d'une simple observation du travail de l'employé et dans une moindre mesure, d'entrevues et de questionnaires spécialisés (Hornsby et Kuratko, 1990). Malheureusement, plus de la moitié des propriétaires-dirigeants entament leur recrutement de personnel sans définir avec exactitude les exigences des emplois à combler. Les conséquences de cette négligence se répercutent bien entendu sur l'ensemble du processus de recrutement et de sélection, mais aussi sur l'évaluation des emplois aux fins de rémunération et de formation.

L'utilisation réelle et le degré de formalisation de l'analyse des emplois (utilisation de formulaires de qualification et d'analyse des emplois) augmentent aussi en fonction de la taille (tableau 5), et plus encore dans les PME syndiquées (Baker, 1955; Hornsby et Kuratko, 1990). En 1969, moins du quart des entreprises (toutes tailles confondues) avaient un programme d'analyse des emplois et seulement un tiers pensaient éventuellement à en établir un (Jones et DeCotiis), cet intérêt variant selon le secteur d'activité. Les raisons les plus souvent mentionnées pour ne pas recourir à ces outils sont les suivantes : aucune utilité justifiable, aucun système acceptable n'a pu être trouvé, trop coûteux, prend trop de temps à réaliser.

\subsubsection{Description de tâches}

Élément le plus connu de l'analyse des emplois, la description de tâches constitue à peu de choses près la seule activité observée en PME pour cette pratique de GRH, son utilisation étant en relation directe avec la taille (Baker, 1955), comme l'indique le tableau 5. De plus, d'Amboise et Gasse (1980), ainsi que McEvoy (1984), rapportent respectivement que $63 \%$ et $35 \%$ de PME ne possèdent aucune description de tâches écrite et que les sources d'information des dirigeants ne sont pas suffisamment spécialisées. 


\section{TABleau 5}

Analyse des emplois en PME

(grandes tendances observées; données tirées de plusieurs études empiriques; par ordre décroissant d'importance)

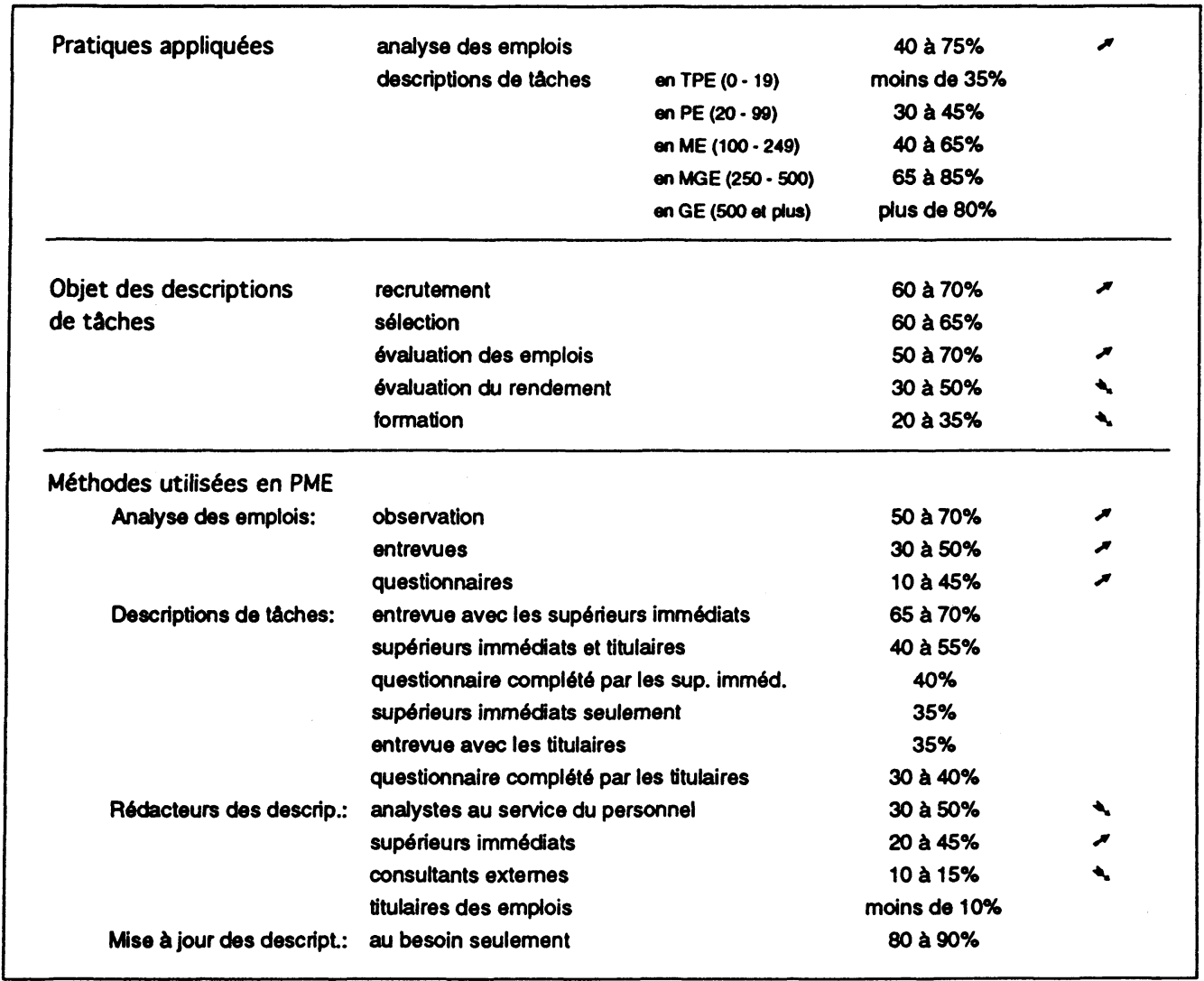


Au contraire, Le Louarn et Thériault (1984), de même que Mahé de Boislandelle et al. (1985), obtiennent des pourcentages assez élevés : plus des trois quarts des PME analysées auraient des définitions de postes tandis que Venet (1988) note que $40 \%$ des entreprises possèdent des descriptions de tâches, dont $30 \%$ des firmes de 10 à 49 salariés; les cadres ayant la leur deux fois plus souvent que les autres catégories occupationnelles. L'utilisation de ces documents varie de l'embauche (présentation de postes) aux affectations et remplacements, à la répartition des tâches, à la rémunération, ainsi qu'à la réflexion sur les fonctions et la recherche de productivité. Enfin, Hornsby et Kuratko (1990) présentent des données plus pondérées : environ $44 \%$ des PME possèdent une description de tâches pour l'ensemble des postes, 15 à $23 \%$, pour deux tiers des postes, et 14 à $28 \%$, pour un tiers seulement.

\section{Recrutement, sélection et accueil}

L'ensemble du processus de recrutement, de sélection et d'accueil constitue l'élément fondamental de toute activité de gestion et de production. En effet, il apparaît impossible de faire fonctionner une entreprise sans au préalable y embaucher des individus qui planifieront, exécuteront et contrôleront les activités de la firme. Que l'on soit en TPE ou en TGE, la dotation revêt en tout temps une importance primordiale pour le succès de l'organisation (Neiswander et al., 1987). Il n'y a aucun doute que le recours à des politiques et procédures formelles de recrutement et de sélection s'accroît directement avec la taille organisationnelle, sous l'unique responsabilité du propriétaire-dirigeant. Entre 30 et $50 \%$ des PE possèdent de tels documents écrits, incluant un simple formulaire, comparativement aux trois quarts des entreprises de moyenne et grande dimensions (Baker, 1955 ; Le Louarn et Thériault, 1984 ; Benoit et Rousseau, 1990).

\subsection{Recrutement}

\subsubsection{Décisions et responsabilités de recrutement}

En TPE, la décision initiale de recrutement incombe souvent au propriétairedirigeant $(35 \%)$ et passe graduellement aux superviseurs à mesure qu'augmente la taille ( $25 \%$ en TPE à $65 \%$ en GE) (Mahé de Boislandelle et al., 1985; Little, 1986; Hillau, 1987). Cependant, plus de 50\% des PME n'entament un recrutement que lorsque cela s'avère absolument nécessaire, après avoir épuisé toutes les autres avenues possibles (tableau 6). Dès cet instant, l'entrepreneur assume lui-même la responsabilité du processus de recrutement (55\% en TPE; $15 \%$ en MGE), en déléguant peu à peu ces tâches à un superviseur, un cadre ou au responsable du personnel lorsque l'entreprise devient plus grande. Par 


\section{TABleau 6}

Décisions et responsabilités de recrutement et sélection en PME

(grandes tendances observées; données tirées de plusieurs études empiriques ; par ordre décroissant d'importance)

\begin{tabular}{|c|c|c|c|c|}
\hline $\begin{array}{l}\text { Politiques écrites } \\
\text { Formulaires utilisés }\end{array}$ & $\begin{array}{l}\text { demande d'emploi } \\
\text { rapport ơentrevue } \\
\text { rapport d'examen médical } \\
\text { réquisition de personnel } \\
\text { demande de référence spéc }\end{array}$ & & $\begin{array}{c}30 \text { a } 75 \% \\
40 \text { a } 90 \% \\
20 \text { a } 40 \% \\
15 \text { a } 55 \% \\
5 \text { a } 30 \% \\
\text { moins de } 25 \%\end{array}$ & 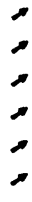 \\
\hline $\begin{array}{l}\text { Décision initiale } \\
\text { de recrutement }\end{array}$ & $\begin{array}{l}\text { supenviseurs } \\
\text { dirigeants } \\
\text { autres cadres } \\
\text { service du personnel }\end{array}$ & & $\begin{array}{l}25 \text { a } 65 \% \\
5 \text { a } 35 \% \\
\text { moins de } 10 \% \\
\text { moins de } 5 \%\end{array}$ & + \\
\hline Détermination des besoins & $\begin{array}{l}\text { brsque nécessaire seuleme } \\
\text { de façon planifiée }\end{array}$ & & $\begin{array}{l}\text { plus de } 50 \% \\
10 \text { \& } 40 \%\end{array}$ & 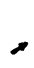 \\
\hline $\begin{array}{l}\text { Responsabilité du processus } \\
\text { de recrutement }\end{array}$ & $\begin{array}{l}\text { propriétaire-dirigeant } \\
\text { responsable de la GRH } \\
\text { PME ayant un dir. du pers.: } \\
\text { PME sans dir. du pers.: }\end{array}$ & $\begin{array}{l}\text { dir. du personnel } \\
\text { cadre } \\
\text { propriétaire-dirigeant } \\
\text { proprietaire-dirigeant } \\
\text { supenviseur }\end{array}$ & $\begin{array}{c}15 \text { à } 55 \% \\
15 \text { à } 45 \% \\
45 \% \\
25 \% \\
15 \% \\
55 \% \\
35 \%\end{array}$ & + \\
\hline $\begin{array}{l}\text { Responsabilité du processus } \\
\text { de sélection }\end{array}$ & $\begin{array}{l}\text { propriétaire-dirigeant } \\
\text { responsable de la GRH } \\
\text { supérieur hiérarchique }\end{array}$ & & $\begin{array}{l}50 \text { à } 60 \% \\
20 \text { à } 30 \% \\
10 \text { à } 15 \%\end{array}$ & $\begin{array}{l}4 \\
+\end{array}$ \\
\hline $\begin{array}{l}\text { Responsabilité de la décision } \\
\text { finale d'embauche }\end{array}$ & $\begin{array}{l}\text { propriétaire-dirigeant } \\
\text { gérant } \\
\text { directeur du personnel + aut } \\
\text { supérieur immédiat } \\
\text { autre intervenant } \\
\text { directeur du personnel seule }\end{array}$ & re cadre & $\begin{array}{c}50 \text { à } 55 \% \\
10 \text { à } 35 \% \\
20 \text { à } 25 \% \\
10 \text { à } 25 \% \\
10 \text { à } 15 \% \\
0 \text { à } 15 \%\end{array}$ & 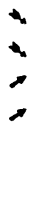 \\
\hline
\end{tabular}


contre, le propriétaire-dirigeant conserve la responsabilité de la sélection (50 à $60 \%$ ) et de la décision finale d'embauche (50 à 55\%) jusqu'à ce qu'il soit secondé par un préposé au personnel qui prend alors la relève avec l'aide d'un cadre ou d'un superviseur.

\section{Sources de recrutement}

Elles s'avèrent beaucoup moins formelles en PME, car on y observe l'existence d'un réseau informel articulé autour du système de relations de l'entrepreneur, assurant ainsi une certaine homogénéité des membres de l'organisation. Les procédures y sont limitées au strict minimum, les échanges sont directs, sans détour, mais le propriétaire-dirigeant hésite généralement à diversifier ses sources de recrutement.

\subsubsection{Sources et méthodes internes}

Même si les PME sont déjà confrontées à une main-d'œuvre réduite, les candidatures recommandées par le personnel, parfois assorties d'un modeste boni s'il y a embauche (McEvoy, 1984), ressortent parmi les trois plus fréquentes sources de recrutement, toutes les autres formes internes étant peu utilisées (tableau 7): affichage interne, transferts, mutations et promotions, anciens employés (Baker, 1955; Le Louarn et Thériault, 1984 ; Mahé de Boislandelle et al., 1985; Venet, 1988). Les recommandations du personnel sont utilisées par $67 \%$ des PE, $75 \%$ des entreprises de 51 à 100 employés et $69 \%$ des ME de 101 à 150 personnes. Elles apparaissent particulièrement importantes en $\mathrm{PE}$, les dirigeants de GE demeurant plus réticents à accepter les suggestions de leur personnel en matière de recrutement, généralement craintifs de voir se créer des groupes au sein de leur entreprise. Comme les outils de sélection sont pratiquement inconnus en PME, les recommandations formulées par des employés dont la performance et l'attitude ont toujours été satisfaisantes permettent en quelque sorte de réduire sensiblement l'incertitude liée au recrutement de nouveaux individus, spécialement dans les plus petites entreprises. Cependant, ce moyen reste essentiellement dépendant du réseau informel de l'entrepreneur, mais procure aussi de l'information sur les salaires et conditions de travail du marché et des compétiteurs. En plus de constituer un moyen économique de recruter des travailleurs, ce réseau assure un certain équilibre, car les individus partagent habituellement une formation et des antécédents comparables (Lépine, 1986).

\subsubsection{Sources et méthodes externes}

Sans contredit les plus courantes dans l'ensemble des entreprises, les méthodes externes les plus fréquemment utilisées en PME sont les candidatures spontanées, les annonces dans les journaux et les contacts personnels du dirigeant 
TABleau 7

Sources de recrutement utilisées en PME

(grandes tendances observées; données tirées de plusieurs études empiriques; par ordre décroissant d'importance)

\begin{tabular}{|c|c|c|c|c|c|c|}
\hline & $1 \%$ & 18 & 4 & $14 \%$ & GP & \\
\hline Recommandations du personnel & 40 à $75 \%$ & 45 à $75 \%$ & 50 à $75 \%$ & 50 à $80 \%$ & 30 à $55 \%$ & + \\
\hline Candidatures spontanées & 35 à $60 \%$ & 45 à $65 \%$ & 50 à $65 \%$ & 60 à $70 \%$ & 75 à $80 \%$ & $\pi$ \\
\hline Annonces dans les joumaux & 25 à $50 \%$ & 30 à $60 \%$ & 50 a $65 \%$ & 40 à $70 \%$ & $70 \%$ & 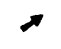 \\
\hline Mutations et promotions & $\ldots \ldots \ldots$ & $\ldots \ldots \ldots$ & 35 à $50 \%$ & $\ldots \ldots \ldots$ & $\ldots \ldots \ldots$ & 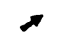 \\
\hline Anciens employés & $\ldots \ldots \ldots$ & $\ldots \ldots \ldots$ & 30 a $45 \%$ & $\ldots \ldots \ldots$ & $\ldots \ldots \ldots$ & + \\
\hline Centres d'emploi gouvernementaux & 20 à $40 \%$ & 20 à $35 \%$ & 25 à $50 \%$ & 30 a $45 \%$ & 25 a $40 \%$ & \\
\hline Institutions d'enseignement & 15 à $20 \%$ & 20 à $25 \%$ & 20 à $35 \%$ & 25 à $40 \%$ & 30 à $45 \%$ & $\Rightarrow$ \\
\hline Agences privées de placement & 10 à $20 \%$ & 15 à $25 \%$ & 20 à $25 \%$ & 25 a $30 \%$ & $30 \%$ & $\lambda$ \\
\hline
\end{tabular}


(tableau 7). Les agences publiques et privées d'emploi forment un second groupe, suivies des institutions d'enseignement (programmes coopératifs, stages, recrutement sur le campus) et des services de placement des associations professionnelles ou syndicales (Baker, 1955; McEvoy, 1984; Le Louarn et Thériault, 1984 ; Hornsby et Kuratko, 1990; Benoit et Rousseau, 1990). Enfin, les PME de moins de 50 employés recourent rarement au conseil externe en recrutement (Mahé de Boislandelle et al., 1985; Venet, 1988) et les médias électroniques (radio, télévision) demeurent encore à des coûts strictement inabordables pour les PME. En outre, une forte proportion de PME québécoises privilégie la banque interne de curriculum vitæ (Audet et al., 1987).

Globalement, les TGE semblent utiliser davantage l'ensemble des sources de recrutement, les plus petites se limitant au premier groupe de méthodes. McEvoy (1984) rapportait initialement que dans les stratégies de recrutement en PME, on dénote peu d'imagination. Étant donné ce manque de créativité, il n'est pas étonnant que les PE déclarent que la recherche de travailleurs compétents soit un problème majeur. Toutefois, Hornsby et Kuratko (1990) nuancent ces propos en signalant que les PME en général utilisent beaucoup plus qu'on ne le pense toutes les méthodes de recrutement, bien que les TPE s'en remettent surtout aux sources les moins coûteuses. Larrivée et d'Amboise (1989) soulignent toutefois que la pratique du recrutement paraît particulièrement cruciale à améliorer et plusieurs techniques peuvent favoriser l'obtention de meilleurs résultats. Ces auteurs suggèrent d'autres méthodes peu coûteuses pouvant être adoptées par les PME: les associations professionnelles; le concept de coopération interentreprises ; l'octroi d'un boni aux employés ayant recommandé un candidat ultérieurement embauché; l'établissement d'un réseau de contacts soutenus avec les écoles.

\subsection{Sélection}

\subsubsection{Décisions et responsabilités de sélection}

Cette responsabilité paraît assez centralisée en PE autour du propriétairedirigeant, tant dans les activités courantes de sélection qu'au moment de la décision finale d'embauche, et en ME au supérieur immédiat, lequel mène les entrevues préliminaire et d'embauche (Little, 1986; Audet et al., 1987). Par contre, plus la taille de l'entreprise augmente, plus la responsabilité de la décision finale d'embauche passe du propriétaire ou du dirigeant au superviseur ou au directeur du personnel, selon une relation mixte avec la taille (Baker, 1955; Gosselin, 1979; Le Louarn et Thériault, 1984). Cette décision est habituellement réservée au seul dirigeant ou au chef du service concerné, et ne semble jamais échoir uniquement au directeur du personnel (Mahé de Boislandelle et al., 1985). 
TABLEAU 8

Modes de sélection utilisés en PME

(grandes tendances observées; données tirées de plusieurs études empiriques; par ordre décroissant d'importance)

\begin{tabular}{|c|c|c|c|c|}
\hline \multirow[t]{5}{*}{ Etude des candidatures } & \multirow{2}{*}{\multicolumn{2}{|c|}{$\begin{array}{l}\text { formulaire de demande d'emploi } \\
\text { curriculum vitæ }\end{array}$}} & 70 à $100 \%$ & $\varnothing$ \\
\hline & & & plus de $80 \%$ & \\
\hline & \multirow[t]{3}{*}{ références: } & demandées & plus de $80 \%$ & $\varnothing$ \\
\hline & & occasionnellement & 50 à $70 \%$ & \\
\hline & & systématiquement & 25 à $40 \%$ & $\pi$ \\
\hline \multirow[t]{9}{*}{ Entrevues de sélection } & \multicolumn{2}{|c|}{ effectuées systématiquement } & 85 à $100 \%$ & $\pi$ \\
\hline & \multirow[t]{5}{*}{ entrevues: } & individuelles & 35 à $50 \%$ & 4 \\
\hline & & en comité & 20 à $30 \%$ & 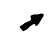 \\
\hline & & individuelles + en comité & 25 à $45 \%$ & $\Rightarrow$ \\
\hline & & semi-structurées & 35 à $55 \%$ & + \\
\hline & & libres / structurées & 25 a $40 \%$ & 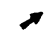 \\
\hline & \multicolumn{2}{|c|}{ nombre moyen: 1 ou 2 par candidat } & 70 à $75 \%$ & \\
\hline & \multirow{2}{*}{\multicolumn{2}{|c|}{ nombre d'entrevues par poste vacant }} & 4 à 5 en PME & \\
\hline & & & plus de 6 en GE & \\
\hline \multirow[t]{6}{*}{ Tests de sélection } & \multicolumn{2}{|c|}{ examen médical (physique) } & 10 à $70 \%$ & 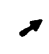 \\
\hline & \multicolumn{2}{|c|}{ aptitudes pratiques / simulation } & 25 à $45 \%$ & 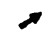 \\
\hline & \multicolumn{2}{|c|}{ intelligence / habiletés mentales } & 20 à $40 \%$ & \\
\hline & \multicolumn{2}{|l|}{ personnalité } & 15 a $30 \%$ & \\
\hline & \multicolumn{2}{|l|}{ intérêts } & 10 à $20 \%$ & \\
\hline & \multicolumn{2}{|c|}{ dépistage des drogues } & moins de $25 \%$ & $\lambda$ \\
\hline
\end{tabular}




\subsubsection{Techniques de sélection}

La majorité des travaux portant sur les techniques de sélection en PME relèvent la prépondérance de trois outils : l'entrevue, le formulaire de demande d'emploi (moindre en TPE) et le curriculum vitæ (tableau 8). Les autres modes semblent plus rares en $P E$ et leur utilisation croît légèrement avec la taille: tests d'aptitudes pratiques, tests psychologiques, formulaires pondérés de demande d'emploi, etc. En PE, le propriétaire-dirigeant connaît bien ses besoins de maind'œuvre et effectue généralement son choix parmi un nombre restreint de candidats. Lorsque l'organisation devient plus grande, le nombre et la complexité des postes augmentent rapidement et le processus de sélection, devenu d'autant plus compliqué, est systématiquement délégué à un subordonné.

D'une part, plus de $80 \%$ des PME possèdent un formulaire de demande d'emploi ou effectuent l'analyse des curriculum vitæ, les proportions s'accroissant directement avec la taille: de 75 à $85 \%$ en TPE jusqu'à 95 et $100 \%$ en ME. D'autre part, plus de $90 \%$ des PME recourent ensuite aux entrevues de sélection, sans distinction cette fois selon la taille (Baker, 1955; McEvoy, 1984; Le Louarn et Thériault, 1984 ; Mahé de Boislandelle et al., 1985; Thacker et Cattaneo, 1987; Hornsby et Kuratko, 1990). De plus, le nombre de curriculum vitæ reçus par poste vacant serait moindre en PME; on y fait surtout des entrevues individuelles $(50 \%)$ et celles-ci s'avèrent moins formelles: $54 \%$ des entrevues étant semi-structurées en PME.

\subsubsection{Références}

Près de $70 \%$ des PME ont recours aux lettres de références, cette utilisation étant moins fréquente en PE (tableau 8), en augmentant légèrement avec la taille. Globalement, les résultats obtenus aux États-Unis et au Québec semblent indiquer une augmentation du taux d'utilisation des lettres de références en PME: Baker parlait de 56 à $74 \%$ en 1955; puis on a noté 71 à $75 \%$ (Le Louarn et Thériault, 1984; Thacker et Cattaneo, 1987); et maintenant, nous relevons 90 à 100\% (Hornsby et Kuratko, 1990). En effet, ces derniers révèlent que $90 \%$ des PE effectuent la vérification des références soumises par les candidats, cette proportion s'élevant à $100 \%$ dans les PME de 51 à 100 employés et à $98 \%$ dans les ME de 101 à 150 personnes. Toutefois, même si les références sont demandées dans la majorité des cas, elles ne sont vérifiées que dans $45 \%$ des entreprises interrogées (Venet, 1988; Benoit et Rousseau, 1990).

\subsubsection{Tests de sélection}

Le recours aux tests dans le processus de sélection en PME varie légèrement selon la taille de l'entreprise (Baker, 1955; McEvoy, 1984; Le Louarn et Thériault, 1984; Thacker et Cattaneo, 1987). Les tests sont principalement 
utilisés par les PME de plus de 101 employés, les PE ne pouvant assumer les coûts rattachés à ces outils de sélection (Hornsby et Kuratko, 1990). De façon très globale (tableau 8), environ un quart des PME utilisent des tests en sélection puisqu'une majorité d'études notent généralement des proportions inférieures à $30 \%$ pour chacun des tests répertoriés. Ainsi, les examens médicaux (physiques), les simulations pratiques (clerical, work samples, trade tests) et la période de probation semblent être régulièrement utilisés. Quant aux autres tests, beaucoup moins fréquents en PME, on retrouve par ordre décroissant les tests d'intelligence, d'aptitudes ou d'habiletés mentales, certains tests psychologiques, soit de personnalité ou d'intérêts, des tests de dépistage des drogues, et en tout dernier lieu, les centres d'évaluation (moins de $20 \%$ ) et l'utilisation du polygraphe (États-Unis seulement: 1 à $2 \%$ ).

\subsubsection{Discrimination en sélection}

Les questions de discrimination en PME ont principalement trait au processus de recrutement et sélection, et de nombreux éléments discriminatoires peuvent être observés dans les formulaires de demande d'emploi (Halatin et al., 1985). L'analyse démontre que $21 \%$ des répondants utilisent des formulaires ne contenant pas de questions «illégales», mais $79 \%$ comportent une ou plusieurs questions mal formulées. En réalité, les PME feraient un usage routinier et constant de propositions illégales ou les utiliseraient de manière discriminatoire, probablement par manque d'expérience ou d'intérêt. Il faut bien reconnaître que plusieurs PME se procurent leurs formulaires dans des entreprises de formules d'affaires ou de fournitures de bureau, en présumant qu'elles respectent les lois et règlements en vigueur, ce qui n'est malheureusement pas toujours le cas.

\subsection{Difficultés du processus de recrutement et sélection}

En PME, il existe à peu près partout des difficultés de recrutement de travailleurs qualifiés, compliquées d'un roulement élevé du personnel qui semble peu considéré par les entrepreneurs (Mahé de Boislandelle et al., 1985; Verser, 1987; Venet, 1988 ; Benoit et Rousseau, 1990). En fait, la majorité des firmes ne dispose pas d'une expertise interne suffisante en gestion et ces lacunes handicapent leur capacité d'attirer du personnel et de le conserver. Larrivée et d'Amboise (1989) viennent confirmer partiellement ces observations en signalant qu'environ $33 \%$ des propriétaires-dirigeants croient que ces difficultés persisteront au cours des cinq prochaines années. Quant aux facteurs explicatifs, ces auteurs mentionnent la capacité de payer des salaires élevés (45\%), la taille de l'entreprise (34\%) et la localisation de la firme (32\%). Des facteurs externes affectent aussi l'existence ou la gravité d'une difficulté de recrutement: dés programmes 
scolaires peu appropriés (49\%), la faiblesse économique du secteur industriel $(45 \%)$ et l'impact de la situation économique générale (35\%). Afin de réduire ou d'éliminer les difficultés de recrutement, $75 \%$ des PME suggèrent de mieux planifier la production, $59 \%$ une formation en entreprise plus appropriée, $59 \%$ la planification formelle des besoins de main-d'œuvre, $45 \%$ préfèrent accroître le travail en temps supplémentaire, $23 \%$ s'en remettent à l'utilisation de la soustraitance, et $22 \%$ proposent l'établissement de contacts avec les écoles.

De la même façon, Laroche (1989) qualifie les difficultés de recrutement de problème principal des RH en PME. Il rapporte que les PE font face à un problème rampant et structurel: la difficulté de recruter de la main-d'œuvre qualifiée, problème qui n'est pas étranger à l'environnement et aux conditions de travail qu'elles offrent, constitue une préoccupation majeure pour un grand nombre de PME et gêne considérablement leur développement. Selon lui, les dirigeants de $\mathrm{PE}$ gagneraient à se poser davantage de questions plutôt que de toujours renvoyer presque mécaniquement le fardeau du problème sur l'offre de main-d'œuvre et sur les institutions, comme ils ont trop tendance à le faire. Ils devraient, par exemple, s'interroger sur la qualité des emplois offerts et les conditions de travail associées à ces emplois, surtout lorsqu'il s'agit de profils professionnels communs aux GE, qui offrent généralement de meilleures conditions.

\subsection{Efficacité du processus de recrutement et validation de la sélection}

Peu d'employeurs effectuent une évaluation de la valeur relative des sources de recrutement utilisées (Baker, 1955; Le Louarn et Thériault, 1984; Mahé de Boislandelle et al., 1985), les deux tiers ne connaissant même pas leurs coûts de recrutement et la très grande majorité des propriétaires-dirigeants ignorant de toute manière comment en vérifier l'efficacité, exception faite de l'appréciation individuelle des performances lors de la période de probation. Toutefois, ces pratiques deviennent plus fréquentes à mesure que la taille augmente, le seuil critique se situant aux alentours de 200 employés.

Ainsi, d'après les perceptions des propriétaires-dirigeants de PME, recueillies sur une échelle Likert en cinq points et regroupées au tableau 9, Hornsby et Kuratko (1990) démontrent que les méthodes de recrutement les plus efficaces en PME sont les suivantes: recommandations des employés, annonces dans les journaux et candidatures spontanées. Ils ajoutent que l'entrevue et les formulaires de demande d'emploi s'avèrent largement plus efficaces que les tests de sélection (tableau 9). En effet, bien que leur efficacité croisse substantiellement en fonction de la taille, passant de 2,61 à 2,81 et à 2,99 (efficacité moyenne des trois catégories de tests, par taille organisationnelle), 
TABleau 9

Efficacité du processus de recrutement et sélection en PME

(grandes tendances observées; données tirées de plusieurs études empiriques ; par ordre décroissant d'importance)

\begin{tabular}{|c|c|c|c|c|}
\hline \multirow{2}{*}{\multicolumn{2}{|c|}{ Délais pour combler un poste vacant }} & moins d'un mois & 45 a $50 \%$ & + \\
\hline & & entre 1 of 2 mois & 30 à $40 \%$ & + \\
\hline \multicolumn{3}{|c|}{ Vérification de l'efficacité du recrutement } & 40 à $65 \%$ & 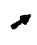 \\
\hline \multirow{2}{*}{\multicolumn{2}{|c|}{ Satisfaction à l'égard des sources de recrutement }} & plutôt satisfait & 60 à $65 \%$ & \\
\hline & & trés satistail & 20 à $30 \%$ & $\nearrow$ \\
\hline \multirow{2}{*}{\multicolumn{2}{|c|}{ Vérification de la qualité des personnes embauchées }} & systématique & 55 à $80 \%$ & 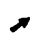 \\
\hline & & informelle & 20 à $35 \%$ & + \\
\hline \multirow{4}{*}{$\begin{array}{l}\text { Validation du processus } \\
\text { de recrutement et sélection : }\end{array}$} & \multicolumn{2}{|l|}{ période de probation } & plus de $75 \%$ & $\varnothing$ \\
\hline & \multicolumn{2}{|l|}{ de façon informelle } & 40 a $70 \%$ & \\
\hline & \multicolumn{2}{|l|}{ aucune validation } & 20 à $50 \%$ & 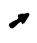 \\
\hline & \multicolumn{2}{|l|}{ de façon formelle } & moins de $15 \%$ & 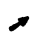 \\
\hline \multirow{6}{*}{$\begin{array}{l}\text { Efficacité des sources } \\
\text { de recrutement } \\
\text { (efficacín moyenne, sur 5) }\end{array}$} & \multirow{2}{*}{\multicolumn{2}{|c|}{ recommandations des employés }} & 3,37 & ת \\
\hline & & & 3,11 & 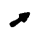 \\
\hline & \multicolumn{2}{|c|}{ candidatures spontanées } & 3,05 & $\Rightarrow$ \\
\hline & \multicolumn{2}{|c|}{ agences privées de placement } & 2,94 & $\pi$ \\
\hline & \multicolumn{2}{|c|}{ centres d'emploi gouvernementaux } & 2,76 & $\leftrightarrow$ \\
\hline & \multicolumn{2}{|l|}{ radio } & 2,58 & $\leftrightarrow$ \\
\hline \multirow{6}{*}{$\begin{array}{l}\text { Efficacité des modes } \\
\text { de sélection } \\
\text { (efficacité moyenne, sur } 5 \text { ) }\end{array}$} & \multirow{2}{*}{\multicolumn{2}{|c|}{ entrevues }} & 4,60 & 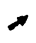 \\
\hline & & & 3,89 & $\pi$ \\
\hline & \multicolumn{2}{|l|}{ vérification des références } & 3,70 & + \\
\hline & \multicolumn{2}{|c|}{ tests d'intelligence et d'aptitudes } & 3,05 & 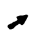 \\
\hline & \multicolumn{2}{|c|}{ tests psychologiques } & 2,72 & 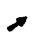 \\
\hline & \multicolumn{2}{|l|}{ tests anti-drogues } & 2,65 & 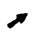 \\
\hline
\end{tabular}


elle demeure nettement au-dessous des autres méthodes de sélection, qui laissent voir des efficacités moyennes de 4,00, 4,04 et 4,15, selon la taille (petite, moyenne ou grande PME). Toutefois, bien qu'il soit moins fréquent, le recours aux tests permet d'accroître l'efficacité du processus de sélection et complète avantageusement l'entrevue traditionnelle (McEvoy, 1984; Thacker et Cattaneo, 1987).

Par ailleurs, bien que $66 \%$ des répondants utilisent une forme ou l'autre de tests en sélection, il apparaît fort préoccupant de noter le peu de connaissances qu'ils ont du concept de validité : entre 40 et $52 \%$ des répondants n'ont jamais entendu parler des trois types de validité (contenu, concomitante et prédictive), la plupart d'entre eux ayant recours à des techniques de sélection sans en connaître la validité (Thacker et Cattaneo, 1987). En effet, près de $90 \%$ des PME ne valident d'aucune façon leurs techniques de sélection (seulement $12 \%$ des répondants ont tenté de le faire empiriquement), ni ne tiennent à jour des fichiers de sélection, ni ne les révisent pour en faire ressortir les prédicteurs de validité ou les effets négatifs sur les minorités. De plus, une faible proportion de PME recourent aux tests d'aptitudes pratiques et aux périodes de probation pour valider la sélection, malgré leur grande efficacité.

\subsection{Accueil}

Cette dernière étape du processus de recrutement et sélection a souvent été peu considérée en PME. En fait, il ne semble pas qu'il y ait absence totale de politiques d'accueil: on parle plutôt de méthodes ou de procédures très légères (Mahé de Boislandelle et al., 1985), majoritairement informelles et peu systématisées (tableau 10). L'accueil et l'intégration se résument ainsi: visite de l'entreprise, programme systématique étalé dans le temps, livret d'accueil et parrainage dans les firmes de plus de 50 employés (Venet, 1988). En fait, une proportion supérieure de PME laisse au superviseur la tâche de présenter l'employé à ses collègues, à son lieu de travail, en lui expliquant parfois les règlements et produits et, dans une moindre mesure, en lui fournissant une aide jusqu'à l'accueil formel de l'individu. Sans véritable formalisation, ces activités s'effectuent souvent sur le tas. Les TPE recourent plus souvent au parrainage (Baker, 1955; Amba-Rao et Pendse, 1982; Benoit et Rousseau, 1990) ou à la période de probation (Audet et al., 1987), et une majorité de PME françaises ne perçoivent pas de besoin systématique pour l'implantation d'un programme formel d'accueil (Mahé de Boislandelle et al., 1985).

Néanmoins, il existe dans de rares PE un programme formel d'accueil qui s'intègre dès l'embauche à une culture organisationnelle dynamique orientée vers la participation de l'employé. Nebenhaus (1987) signale en PE l'existence 
TABleau 10

Modalités d'accueil en PME

(grandes tendances observées; données tirées de plusieurs études empiriques; par ordre décroissant d'importance)

Politiques écrites

moins de $35 \%$

Procédures établies

plus de $70 \%$

Méthodes et pratiques (pourcentages très largement dispersés; par ordre décroissant ơimportance)

$1^{\circ}$ présentation, visite de l'entreprise; accueil des nouveaux employés à leur lieu de travail

$2^{\circ}$ parrainage continu des nouveaux employés

$3^{\circ}$ programme systématique étalé dans le temps

$4^{\circ}$ livret d'accueil, vidéo, programmes-maison, etc.

Information fournie aux employés

réglementation interne du travail

syndicat

produits et services de l'entreprise

assurance-retraite

structures organisationnelles

sports et loisirs

copie des politiques de GRH

75 à $95 \%$

70 à $80 \%$

60 à $70 \%$

35 aे $90 \%$

30 aे $40 \%$

moins de $30 \%$

moins de $20 \%$ 
d'un dossier d'accueil complet, et bien qu'il soit exceptionnel, celui-ci est véritablement tenu à jour et son contenu éloquent confirme l'importance du discours sur la participation et la volonté de plonger d'emblée le nouvel arrivant dans cet état d'esprit. Ternon (1989) a aussi remarqué dans des PME innovantes qu'une bonne partie de la mobilisation des employés se joue à l'accueil. Faudrait-il croire que les PME qui ignorent cette pratique n'ont pas de vision à partager avec leur personnel? Peut-être ignorent-elles simplement les avantages d'un accueil mieux orchestré, ce qui parait plausible dans le contexte d'urgence lié à l'embauche de main-d'œuvre dans plusieurs PME.

\section{Conclusion}

La présente démarche de recension critique de la documentation spécialisée a permis de répondre principalement aux questions suivantes:

- Quelles sont les pratiques d'acquisition des RH appliquées dans les PME, et dans quelle mesure?

- Quel est leur degré de formalisation?

- Quelle influence a cette formalisation sur l'existence et l'étendue de ces pratiques?

Afin d'apporter une réponse satisfaisante à ces interrogations au-delà des préjugés, des opinions, des perceptions ou même des expériences personnelles nécessairement limitées, ce diagnostic appelait une analyse de l'expérience internationale à travers la documentation empirique consacrée aux pratiques d'acquisition des RH en PME. Pour être retenue dans notre synthèse, chacune des études devait respecter les critères scientifiques des enquêtes, à la composition de leur échantillon, ainsi qu'à l'utilisation d'au moins un facteur de contingence dans l'interprétation de leurs résultats empiriques. Quant à notre méthode d'analyse, elle s'apparentait à l'analyse de contenu dans la mesure où les données empiriques des études ont été découpées en unités significatives associées aux pratiques de GRH.

Les résultats semblent justifier certaines réponses aux questions de recherche originales, en démontrant qu'il existe réellement des pratiques d'acquisition des RH en PME. Bien qu'elles demeurent généralement peu formalisées, cellesci existent à des degrés fort variables, démontrant le caractère fortement hétérogène des entreprises de petites et moyennes dimensions, interdisant dès lors toute autre forme de généralisation. En effet, la mise en œuvre de ces pratiques dépend aussi du contexte légal et des contraintes organisationnelles : plusieurs pratiques sont régies par des réglementations nationales en France, et bien que ces normes 
légales ne soient pas systématiquement appliquées en PME, elles exercent une influence notable sur les pratiques réelles de GRH. Par contre, à l'autre extrême, on observe en Amérique du Nord une présence réglementaire moins étendue en PME.

L'analyse du degré de formalisation des pratiques d'acquisition des $\mathrm{RH}$ en PME permet de constater l'existence de différences substantielles dans leur mise en application selon la taille des entreprises, jusqu'à un plateau variant entre 400 et 600 employés. À ce stade, on retrouve la majorité des pratiques, sous la gouverne d'une équipe de professionnels de la GRH et dans un contexte beaucoup plus élaboré qu'en TPE ou en PE. En effet, très peu de PE effectuent une réelle planification des $\mathrm{RH}$ ou une véritable analyse des emplois : quelquesunes établissent un plan de recrutement à court terme et mettent sur papier des descriptions de tâches, mais ces outils ne servent qu'à se conformer à des normes provenant de l'extérieur (règlements, subventions, aides à l'emploi). La majorité des PME démontrent peu d'imagination dans le choix des sources de recrutement et se bornent aux candidatures spontanées, aux recommandations du personnel et aux annonces classées des journaux. La sélection demeure au stade primaire en se limitant à l'analyse des formulaires de demande d'emploi et aux entrevues individuelles: on a recours à très peu de critères de sélection, les tests apparaissent sous-utilisés et le processus d'accueil ne dépasse guère la présentation aux collègues de travail.

La présente recherche laisse entrevoir certaines limites dont il faut tenir compte dans l'appréciation des résultats qui en découlent. En premier lieu, cet examen de la documentation ne peut prétendre à l'exhaustivité. D'abord, parce que même si l'analyse impliquait au-delà de 100 périodiques publiés dans une douzaine de pays, elle se limitait tout de même à la documentation empirique en langues française et anglaise de quelques pays occidentaux. Ensuite, parce que parmi cette dernière, il y a possiblement eu des omissions involontaires devant la panoplie de publications scolaires et professionnelles diffusées sur les plans local et régional. Une seconde limite réside dans l'hétérogénéité des apports méthodologiques de chaque étude empirique. En effet, on doit reconnaître la difficulté inhérente à la synthèse de résultats empiriques dont la qualité méthodologique s'avère fortement hétérogène, entraînant un risque élevé associé à la généralisation des données statistiques présentées. Par conséquent, l'élaboration de certains tableaux-synthèses nous a obligés à tracer des tendances médianes excluant certains résultats marginaux qui correspondaient parfois à la situation réelle des pratiques de GRH dans plusieurs PME.

Ces considérations incitent à formuler certaines recommandations aux auteurs désireux de poursuivre des recherches portant sur les pratiques de GRH en PME, recherches que l'on souhaiterait de meilleure qualité méthodologique 
et conceptuelle. L'adaptation de la recherche aux besoins réels des PME en matière de GRH devrait s'appuyer sur une base d'information plus étoffée et ajustée aux pratiques réelles des PME, à partir d'études empiriques ciblées et menées dans des secteurs d'activité comparables et homogènes, sur des échantillons d'entreprises de même taille. En fait, la probabilité d'obtenir des résultats peu significatifs demeure très élevée dans ce genre d'analyse: la trop forte diversité des entreprises enquêtées peut grandement réduire la qualité de l'interprétation des données recueillies. Par ailleurs, une autre précaution méthodologique pourrait résider dans le recours aux entrevues directes, de préférence à l'envoi de questionnaires par la poste, cette dernière méthode de collecte de données se révélant peu adaptée aux caractéristiques des propriétaires-dirigeants de PME.

Par ailleurs, une autre série de questions pertinentes concerne les pistes de solutions inexplorées en GRH. Étant donné les particularités de leur environnement, les PME auraient-elles avantage à développer ou à adapter leurs pratiques d'acquisition des RH? Dans une telle perspective, on pourrait suggérer des adaptations innovatrices de pratiques existantes ou carrément l'élaboration de pratiques nouvelles qui répondraient mieux aux contraintes inhérentes au contexte des PME. Cette démarche ne consisterait plus seulement à expliquer l'existant en GRH, mais également à identifier le souhaitable. Par analogie avec l'évolution des connaissances relatives aux grandes entreprises, on pourrait envisager ici des solutions s'inscrivant dans de nouveaux paradigmes de gestion, de la même façon que le mouvement de gestion participative est venu ébranler et remettre en question le paradigme taylorien de l'organisation scientifique du travail.

Dans cette optique, il semble essentiel d'orienter d'éventuelles démarches de recherche vers une vérification empirique de l'existence de possibles relations entre le degré de formalisation de la GRH et le secteur d'activité, les ressources financières et matérielles de l'entreprise, la présence d'un syndicat, le système de production de la PME ou la vision entrepreneuriale.

En effet, une dernière avenue de recherche apparaît des plus attrayantes: l'évaluation des impacts potentiellement significatifs de la vision entrepreneuriale sur l'existence et la formalisation de la GRH en PME. Notre analyse a permis de pressentir la priorité devant être accordée à l'entrepreneur quant à l'ouverture de sa vision vers l'intégration et la délégation de la GRH dans l'organisation. Souvent, la stratégie n'existe qu'au niveau de la vision de l'entrepreneur, et bien qu'elle soit traduite en décisions et en actions concrètes au sein de l'organisation, elle demeure informelle et plus ou moins consciente en matière de GRH. En fait, il existe un nombre impressionnant de petites entreprises où la vision de l'entrepreneur se traduit très tôt en politiques et activités 
concrètes de GRH adaptées à son milieu organisationnel et en parfaite harmonie avec tous les membres de l'entreprise. Ces exemples patents de GRH «à vision élevée » auraient avantage à être étudiés et connus plus largement du public. La masse des entrepreneurs traditionnels pourraient alors s'en inspirer d'heureuse façon, ouvrant la voie à une GRH renouvelée dans les organisations de petites et moyennes dimensions.

Finalement, il faut bien comprendre que la formalisation ne constitue pas une nécessité absolue en PME. Elle peut parfois s'avérer utile, mais elle peut être aussi carrément nuisible au bon fonctionnement et à la performance réelle de l'organisation. Il n'y a en réalité aucune désirabilité sociale ni administrative pour la PME à formaliser ses pratiques de gestion. Il faut plutôt considérer la formalisation comme une façon d'opérationnaliser la mesure d'une variable dépendante, en l'occurrence, les pratiques de GRH en PME, et plusieurs indicateurs permettraient d'évaluer le degré de formalisation de la GRH dans une organisation, quelle que soit sa taille organisationnelle (Fabi, Garand et Pettersen, 1993).

\section{Bibliographie}

AMBA-RAO, S.C. et D. PENDSE (1985), «Human resource compensation and maintenance practices », American Journal of Small Business, vol. 10, $n^{\circ} 2$, p. 19-29.

AUdet, M., L. BÉLANGER et F. JACQUES (1987), Place, importance et pratiques de la gestion des ressources humaines dans les entreprises québécoises, Document de recherche, collection Instruments de travail, Département des relations industrielles, Université Laval, Québec, 105 p.

BAKer, A.W. (1955), Personnel Management in Small Plants: A Study of Small Manufacturing Establishments in Ohio, Bureau of Business Research, College of Commerce and Administration, The Ohio State University, Colombus, 288 p.

BAYAD, M. et J.-L. HERRMANN (1991), «Gestion des effectifs et caractéristiques des petites et moyennes entreprises industrielles: vers quelles relations?», Revue Internationale P.M.E., vol. $4, \mathrm{n}^{\circ} 2$, p. 5-41.

BAYAD, M. et J.-L. HerRmANn (1992), « Panel des PMI Lorraines : rapport technique de la troisième vague. Enquête $1991 »$, Centre régional de documentation en gestion, Nancy, janvier.

BENOIT, C. et M.-D. Rousseau (1990), «La gestion des ressources humaines dans les petites et moyennes entreprises au Québec», Revue Internationale P.M.E., vol. $3, \mathrm{n}^{\circ} 1$, p. $39-55$. 
BENOIT, C. et M.-D. RousseAu (1993), La gestion des ressources humaines dans les $P M E$ au Québec: perception des dirigeants, pour le ministère de la Maind'œuvre, de la Sécurité du revenu et de la Formation professionnelle, Direction de la recherche, Publications du Québec, 293 p.

CATHERINE, J. (1990), «La fonction personnel dans les établissements ou entreprises de 200 à 2000 personnes », Les Nouveaux Cahiers ANDCP, Paris, $\mathrm{n}^{\circ}$ 40, p. 17-34.

D'AMBOISE, G. et A. PARENT (1989), Les problèmes des petites entreprises : ce que révèlent des études nord-américaines, Document de travail, Faculté des sciences de l'administration, Université Laval, Québec, $\mathrm{n}^{\circ}$ 89-07.

D'AMBOISE, G. et D.J. GARAND (1993), Identification des difficultés et besoins des PME en matière de GRH, Rapport inédit, pour la Société québécoise de développement de la main-d'œuvre (SQDM), Montréal, octobre, 125 p.

D'Amboise, G. et Y. GASSE (1980), Performance in Small Firms and the Utilization of Formal Management Techniques, Document spécial, Faculté des sciences de l'administration, Université Laval, Québec, $\mathrm{n}^{\circ}$ 80-17.

D'Amboise, G. et Y. GASSE (1982), Managerial Difficulties as Perceived by Small Business Owner-Managers: Where Are the Similarities and Differences?, Document de travail, Faculté des sciences de l'administration, Université Laval, Québec, $n^{\circ}$ 82-13 A.

FABI, B. (1991), «Les facteurs de contingence des cercles de qualité : une synthèse de la documentation empirique ", Revue Canadienne des Sciences de l'Administration, vol. $8, \mathrm{n}^{\circ} 3$, p. 161-174.

FABI, B. et D.J. GARAND (1989), «À propos de l'ouvrage d'Henri Mahé de Boislandelle: "Gestion des ressources humaines dans les P.M.E." ", Revue Internationale P.M.E., vol. 2, n 1, p. 99-108.

FABI, B. et D.J. GARAND (1993), «La gestion des ressources humaines en PME», dans P.-A. Julien (dir.), Les PME: bilan et perspectives, Québec, Les Presses Inter Universitaires, et Paris, Economica.

FABI, B., D.J. GARAND et N. PETTERSEN (1993), «La gestion des ressources humaines en PME: proposition d'un modèle de contingence», dans M. Amami et al., (dir.), Compétitivité des PME et marchés sans frontière, Congrès international francophone de la PME, Carthage, Tunisie, 28-30 octobre, p. 216-229.

GARAND, D.J. (1992), «Synthèse des pratiques d'acquisition des ressources humaines en PME », dans P. Louart et al. (dir.), La GRH avec ou sans frontières, Actes du $3^{\mathrm{e}}$ Congrès de l'AGRH, Lille, p. 412-425.

GARAND, D.J. (1993), Les pratiques de gestion des ressources humaines (GRH) en petites et moyennes entreprises (PME): une synthèse conceptuelle et empirique, Rapport de recherche, Groupe de recherche en économie et gestion des PME (GREPME), mars, 386 p. 
GARAND, D.J. et B. FABI (1991), «Fondements conceptuels des pratiques de GRH en petites et moyennes entreprises (PME): formalisation, vision entrepreneuriale et modèle contingentiel », dans A. BERNARD et al. (dir.), Pour une vision de la GRH, Actes du $2^{\mathrm{e}}$ Congrès de l'AGRH, Cergy Pontoise, p. 324-336.

GARAND, D.J. et B. FABI (1992), «État de la recherche. Les pratiques de gestion des ressources humaines (GRH) en PME», Revue Organisation, vol. 2, $\mathrm{n}^{\circ} 1$, automne, p. 61-99.

GARAND, D.J. et B. FABI (1993), «Les pratiques de conservation des ressources humaines en PME », dans G. TREPO et al. (dir.), Universalité et contingence de la GRH, Actes du 4e Congrès de l'AGRH, HEC-Paris, Jouy-en-Josas, p. 506-523.

Gosselin, L. (1979), La fonction personnel dans l'entreprise québécoise, aspect global et aspect particulier: les perceptions que les directeurs généraux et les directeurs de personnel s'en font, Thèse inédite (M.A.) en relations industrielles, Université Laval, Québec, 156 p.

Halatin, T., J. RoY et B. MidDlebrooK (1985), «The application form in the small business : design and legal considerations », dans J.E. BROTHERS (dir.), The Challenge of Small Business Management Assistance Unification, Comptes rendus de la neuvième conférence annuelle de la SBIDA, San Diego, p. 280-283.

Herman, J. et P. DE Bruyne (1982), L'esprit d'entreprise dans les P.M.I.. Les P.M.I. wallones et leurs dirigeants, Centre d'études praxéologiques de Louvain, Belgique.

HESS, D. (1987), La gestion préventive de l'emploi en PMI, Les cahiers de la recherche, Méthodes - études, I.A.E. de Lille, $\mathrm{n}^{\circ} 87 / 11,26 \mathrm{p}$.

HiLlaU, B. (1987), «PME, gestion du personnel et environnement de l'entreprise», Formation Emploi, $\mathrm{n}^{\circ} 19$, p. 19-24.

HOFFMAN, K. (1986), "Opinions of small Kansas business owners / operators concerning hiring, training, and advancement of male / female non-traditional workers in Kansas », dans H. BUCKMAN STEPHENSON (dir.), The Impact of the Eighties on Small Business: Trends and Issues, Comptes rendus de la dixième conférence annuelle de la SBIDA, Washington, p. 261-266.

Holley, W.H. JR. et R.S. WolTERS (1987), «Employment at-will: An emerging issue for small businesses », Journal of Small Business Management, vol. $25, n^{\circ} 4$, p. $1-8$.

HORNSBY, J.S. et D.F. KURATKO (1990), «Human resource management in small business : critical issues for the 1990's », Journal of Small Business Management, vol. $28, \mathrm{n}^{\circ} 3$, juillet, p. 9-18.

JONES, J.J. et T.A. DECoTIS (1969), «Job analysis : national survey findings », Personnel Journal, vol. 48, $\mathrm{n}^{\circ} 10$, p. 805-809. 
LAROCHE, G. (1989), Petites et moyennes entreprises au Québec : organisation économique, croissance de l'emploi et qualité de vie au travail, Série de recherche $\mathrm{n}^{\circ}$ 91, Institut international d'études sociales, Genève, $84 \mathrm{p}$.

LARRIVÉE, L. et G. D’ AMBoISE (1989), « Difficultés de recrutement dans les P.M.E. québécoises : quelques causes et solutions possibles », Relations Industrielles, vol. $44, \mathrm{n}^{\circ} 3$, p. 487-506.

LE LOUARN, J.-Y. et R. THÉRIAULT (1984), Politiques et pratiques en recrutement et sélection en personnel dans les organisations au Québec, Association des professionnels en ressources humaines du Québec (APRHQ), HEC, Montréal, 79 p.

LÉPINE, I. (1986), « Recruiting practices in a tight labor market», dans A. ANDIAPPAN (dir.), Rapport du Congrès annuel du Groupe d'intérêt particulier sur le personnel et les ressources humaines de l'Association des sciences administratives du Canada (ASAC), Whistler, p. 41-49.

LITTLE, B.L. (1986), «The performance of personnel duties in small Lousiana firms : a research note », Journal of Small Business Management, vol. 24, $\mathrm{n}^{\circ} 4$, p. 66-69.

MAHÉ DE BoIsLANDELle, H. (1988), Gestion des ressources humaines dans les P.M.E., Paris, Economica, 322 p.

MAHÉ DE Boislandelle, H. (1990), «Repérage de la fonction ressources humaines en P.M.E. », Revue Internationale P.M.E., vol. 3, n 1, p. 27-37.

MAhÉ DE Boislandelle, H. (1993), Gérer les hommes de la jeune entreprise, Paris, Chotard Éditeurs, 200 p.

MAHÉ DE Boislandelle, H. et al. (1985), «La pratique de la gestion du personnel dans les P.M.E. », Rapport de recherche, CEGERH / FNEGE / IUT - Montpellier, $150 \mathrm{p}$.

MARLOW, S. et D. PATTON (1992), «Employment relations, human resource management strategies and the smaller firm », Communication à la quinzième conférence sur la recherche et les politiques sur les PME, Southampton, 25-26 novembre.

MCEvoY, G.M. (1984), «Small business personnel practices», Journal of Small Business Management, vol. 22, $\mathrm{n}^{\circ} 4$, p. 1-8.

MealieA, L.W. et D. LeE (1980), «Contemporary personnel practices in Canadian firms : an empirical evaluation », Relations Industrielles, vol. 35, $\mathrm{n}^{\circ} 3$, p. 410-421.

NARO, G. (1990), «Les PME face à la gestion de leurs effectifs : comment adapter les ressources humaines aux impératifs stratégiques? », Revue Internationale P.M.E., vol. $3, \mathrm{n}^{\circ} 1$, p. $57-74$.

NeIswander, D.K., B.J. BIRD et P.L. Young (1990), «Embauche et gestion des premiers employés d'une petite entreprise», Revue Internationale P.M.E., vol. 3, $\mathrm{n}^{\circ} 1$, p. 7-25. 
PAUMIER, A. et D. GouADAIN (1984), «La gestion prévisionnelle des ressources humaines : une enquête auprès d'un échantillon d'entreprises régionales », Direction et Gestion, $\mathrm{n}^{\circ}$ 6, p. 53-68.

RAM, M. (1991), «The dynamics of workplace relations in small firms », International Small Business Journal, vol. 10, $\mathrm{n}^{\circ}$ 1, p. 44-53.

RINDT, K.E. (1974), Use of Employment Agencies by Small Business, University of Wisconsin Extension, Madison.

ROBINSON, D.D. (1981), «Content-oriented personnel selection in a small business setting», Personnel Psychology, vol. 34, $\mathrm{n}^{\circ}$ 1, p. 77-87.

SCOTT, M.G., I. RoberTs, G. Holroyd et D. SAWBRIDGE (1990), Management and Industrial Relations in Small Firms, Cahier de recherche, UK Department of Employment, London, $\mathrm{n}^{\circ} 70$.

TERNON, S. (1989), La gestion des ressources humaines dans les PME innovantes. Enquête auprès de 22 PME de l'Hérault, Étude inédite en Maîtrise de psychologie sociale, Montpellier, $62 \mathrm{p}$.

ThaCKeR, J.W. et R.J. CATTANeo (1987), «The Canadian personnel function: Status and practices », dans T.H. STONE et J.-Y. LE LOUARN (dir.), Rapport du Congrès annuel du Groupe d'intérêt particulier sur le Personnel et les ressources humaines de l'Association des sciences administratives du Canada (ASAC), Toronto, p. 56-66.

VENET, P. (1988), «La fonction personnel dans les P.M.E. des Alpes-Maritimes et de Monaco », Personnel (Revue ANDCP), Paris, $\mathrm{n}^{\circ} 299$, p. 17-21.

VERMOT-GAUD, C. (1987), Prévoir l'emploi et gérer les carrières individuelles, Paris, Éditions d'Organisation.

VERSER, T.G. (1987), « Responses of inexperienced small business owners to perceived personnel problems », dans R.G. WYCKHAM, L.N. MEREDITH et G.R. BUSHE (dir.), The Spirit of Entrepreneurship, Comptes rendus de la trente-deuxième conférence annuelle du Congrès international de la petite entreprise, Vancouver, p. 287-296. 